\title{
Chiral [2.2.2] Dienes as Ligands for $R h(I)$ in Conjugate Additions of Boronic Acids to a Wide Range of Acceptors
}

Christian Defieber, Jean-François Paquin, Sonia Serna, and Erick M. Carreira

The following includes general experimental procedures, specific details for representative reactions, and isolation and spectroscopic information for the new compounds prepared. All reactions were performed in oven dried glass ware under argon. For the reactions, solvents were purified by distillation and dried by passage over activated alumina under an argon atmosphere $\left(\mathrm{H}_{2} \mathrm{O}\right.$ content < 30 ppm, Karl-Fischer titration). 2-Cyclohexenone and 2cyclopentenone were distilled before used. Other commercially available chemicals were used as received unless noted otherwise. ${ }^{1} \mathrm{H}$ and ${ }^{13} \mathrm{C}$ NMR spectra were recorded on a VARIAN Mercury $300 \mathrm{MHz}$ or a Gemini $300 \mathrm{MHz}$. Infrared spectra were recorded on a Perkin-Elmer spectrum RX-I FT-IR or a Perkin Elmer spectrometer. High resolution mass spectra were obtained on a VG-TRIBRID for electron impact ionization (EI) or on a TSQ 7000 for electron-spray ionization (ESI). Combustion analyses were performed on a 
Perkin Elmer AAnalyst 800 or on a Varian SpectraAA 400 Zeemann. Enantiomeric excesses were determined by chiral HPLC analysis with Merck-Hitachi D-7000 system. Optical rotation $[\alpha]_{D}$ were measured on a Jasco DID-1000 Polarimeter. The absolute configurations were assigned by comparison of the $[\alpha]_{D}$ values of known compounds. For the new adducts, it was assigned based on the established stereochemical outcome of the reaction.

\section{Ligand Synthesis}

The following includes the experimental details for the synthesis of the ligands as exemplified for ligand 1.

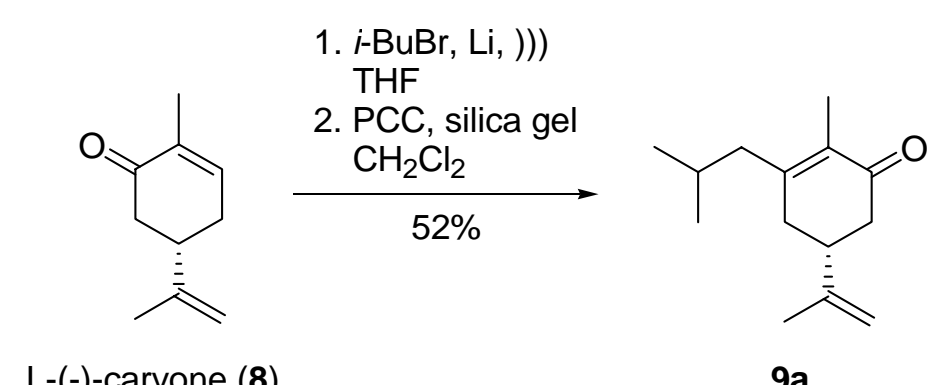

\section{(5R)-3-isobutyl-5-isopropenyl-2-methyl-2-cyclohexen-1-one}

(9a).

A solution of $\mathrm{L}-(-)$-carvone $(3.13 \mathrm{~mL}, 19.9 \mathrm{mmol})$, Li (693 mg, $99.8 \mathrm{mmol})$, iso-butylbromide (8.69 mL, $79.9 \mathrm{mmol})$ in $\operatorname{THF}(150 \mathrm{~mL})$ was sonicated for $1.5 \mathrm{~h}$. The reaction 
mixture was cooled to $0{ }^{\circ} \mathrm{C}$ and quenched slowly with a saturated aqueous $\mathrm{NH}_{4} \mathrm{Cl}$ solution followed by cold water. The THF was removed and the residual aqueous layer was extracted with $\mathrm{Et}_{2} \mathrm{O}(3 \times)$. The combined organic layers were washed with brine, dried over $\mathrm{Na}_{2} \mathrm{SO}_{4}$, and the solvent was evaporated. The crude alcohol was used for the next step without further purification. To a suspension/solution of PCC $(8.40 \mathrm{~g}, 38.9 \mathrm{mmol})$ and silica gel $(8.40 \mathrm{~g})$ in $\mathrm{CH}_{2} \mathrm{Cl}_{2}$ (35 mL) was added a solution the crude alcohol in $\mathrm{CH}_{2} \mathrm{Cl}_{2}$ (35 mL). After stirring for $3 \mathrm{~h}$, the reaction mixture was filtered through a pad of silica gel using $\mathrm{CH}_{2} \mathrm{Cl}_{2}$ as eluent to give the crude product. The latter was purified by flash chromatography using EtoAc/hexane (10:1) to give 9a (2.15 9, 52\% from L-(-)-carvone) as a colorless oil. [ $\alpha]_{\mathrm{D}}^{27} 59.7$ ( $\boldsymbol{C}$ 0.95, $\left.\mathrm{CHCl}_{3}\right) ; \operatorname{IR~(neat)~} v=2956,2869,1667,1627,1463$, 1381, 1165, 1129, 1081, $891 \mathrm{~cm}^{-1} ;{ }^{1} \mathrm{H} \mathrm{NMR}\left(300 \mathrm{MHz}, \mathrm{CDCl}_{3}\right) \delta$ 4.76-4.72 (m, 2H), 2.57-2.50 (m, 2H), 2.33-2.24 (m, 2H), $2.17-2.14(\mathrm{~m}, 1 \mathrm{H}), 1.94-1.81(\mathrm{~m}, 1 \mathrm{H}), 1.74(\mathrm{~s}, 3 \mathrm{H}), 1.71$ $(\mathrm{s}, 3 \mathrm{H}), 0.91(\mathrm{~d}, 3 \mathrm{H}, \boldsymbol{J}=6.6 \mathrm{~Hz}), 0.88(\mathrm{~d}, 3 \mathrm{H}, \boldsymbol{J}=6.6$ $\mathrm{Hz}) ;{ }^{13} \mathrm{C} \mathrm{NMR}\left(75 \mathrm{MHz}, \mathrm{CDCl}_{3}\right) \delta 199.1,157.1,146.7,131.1$, $110.1,44.3,42.7,41.5,36.5,27.5,23.0,22.5,20.6$, 11.2; Anal. Calcd for $\mathrm{C}_{14} \mathrm{H}_{22} \mathrm{O}: \mathrm{C}, 81.50$; $\mathrm{H}, 10.75$. Found: C, 
81.24; $\mathrm{H}$, 10.67. HRMS-EI calcd for $\mathrm{C}_{14} \mathrm{H}_{22} \mathrm{O}[\mathrm{M}]^{+} 206.1671$, found 206.1666 .

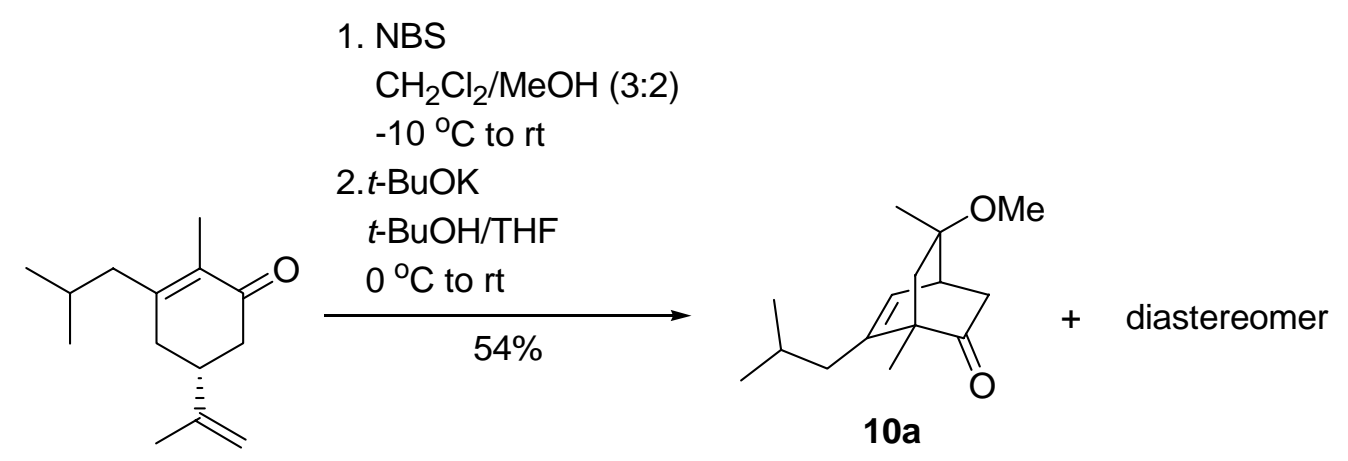

$9 a$

\section{$(1 S, 4 S, 8 R)-6$ - isobutyl-8 - methoxy -1, 8 -}

\section{dimethylbicyclo[2.2.2] oct-5-en-2-one (11a)}

To a solution of $\mathbf{9 a}\left(2.11 \mathrm{~g}, 10.2 \mathrm{mmol}\right.$ ) in $\mathrm{CH}_{2} \mathrm{Cl}_{2}$ (11 $\mathrm{mL})$ and $\mathrm{MeOH}(7 \mathrm{~mL})$ at $-10{ }^{\circ} \mathrm{C}$ was added NBS $(2.18 \mathrm{~g}, 12.3$ mmol) in small portions over 1 h and the reaction was allowed to warm to room temperature overnight. The reaction was diluted with $\mathrm{CH}_{2} \mathrm{Cl}_{2}$ and washed with $1 \mathrm{M}$ aqueous $\mathrm{NaOH}$. The aqueous layer was extracted with $\mathrm{CH}_{2} \mathrm{Cl}_{2}(3 \times)$, the combined organic layers washed with brine, dried over $\mathrm{Na}_{2} \mathrm{SO}_{4}$, and the solvent evaporated. The crude product was purified by flash chromatography using EtOAc/hexane (85:15) to give the intermediate bromo compound $(2.17 \mathrm{~g}, 68 \%)$ as a colorless oil. IR (neat) $v=2955,2688,1663,1629,1459 \mathrm{~cm}^{-1} ;{ }^{1} \mathrm{H}$ NMR 
$\left(300 \mathrm{MHz}, \mathrm{CDCl}_{3}\right) \delta 3.48-3.37(\mathrm{~m}, 2 \mathrm{H}), 3.21(\mathrm{~m}, 3 \mathrm{H}), 2.58-$ $2.08(\mathrm{~m}, 7 \mathrm{H}), 2.00-1.83(\mathrm{~m}, 1 \mathrm{H}), 1.74(\mathrm{~s}, 3 \mathrm{H}) ;{ }^{13} \mathrm{C} \mathrm{NMR}(75$ $\left.\mathrm{MHz}, \mathrm{CDCl}_{3}\right) \delta 199.2,199.0,158.0,156.9,131.3,131.1$, $75.9,75.7,49.5,49.4,44.4,44.3,40.0,39.9,38.7,37.9$, $36.7,36.5,32.1,31.2,27.4$ (2), 22.9 (2), 22.3, 18.1, 17.8, 11.0. To a solution of $t$-BuOK (1.18 g, 10.5 mmol) in $t-\mathrm{BuOH}(10.5 \mathrm{~mL})$ at $0{ }^{\circ} \mathrm{C}$ was added a solution of the bromo compound in THF (11 mL). The reaction mixture was warmed to room temperature and stirred $28 \mathrm{~h} . \mathrm{Et}_{2} \mathrm{O}(75 \mathrm{~mL})$ was added and the solution was washed with $0.5 \mathrm{M}$ aqueous $\operatorname{HCl}(3 \times)$, brine, dried over $\mathrm{Na}_{2} \mathrm{SO}_{4}$, and the solvent was evaporated. Purification of the crude by flash chromatography using EtoAc/hexane (5:1) gave 11a (336 mg), dia-11a (133 mg) along with mixed fraction (845 mg), as colorless oils (1.31 $9,79 \%) \cdot[\alpha]_{\mathrm{D}}^{28} 320.76\left(\boldsymbol{c} 1.37, \mathrm{CHCl}_{3}\right) ; \mathrm{IR}$ (neat) $v=2954$, $2869,2826,1722,1464,1368,1144,1083,1068 \mathrm{~cm}^{-1} ;{ }^{1} \mathrm{H}$ NMR $\left(300 \mathrm{MHz}, \mathrm{CDCl}_{3}\right) \delta 6.04(\mathrm{~d}, 1 \mathrm{H}, \boldsymbol{J}=6.9 \mathrm{~Hz}), 3.14(\mathrm{~s}, 3 \mathrm{H})$, $2.84-2.80(\mathrm{~m}, 1 \mathrm{H}), 2.50(\mathrm{dd}, 1 \mathrm{H}, \boldsymbol{J}=18.1,2.0 \mathrm{~Hz}), 1.93-$ $1.76(\mathrm{~m}, 3 \mathrm{H}), 1.70(\mathrm{~d}, 1 \mathrm{H}, \boldsymbol{J}=13.8 \mathrm{~Hz}), 1.65-1.54(\mathrm{~m}, 1 \mathrm{H})$, $1.43(\mathrm{~d}, 1 \mathrm{H}, \boldsymbol{J}=13.8 \mathrm{~Hz}), 1.23(\mathrm{~s}, 3 \mathrm{H}), 1.10(\mathrm{~s}, 3 \mathrm{H}), 0.88$ $(\mathrm{d}, 3 \mathrm{H}, \boldsymbol{J}=6.5 \mathrm{~Hz}), 0.79(\mathrm{~d}, 3 \mathrm{H}, \boldsymbol{J}=6.5 \mathrm{~Hz}) ;{ }^{13} \mathrm{C} \operatorname{NMR}(75$ $\left.\mathrm{MHz}, \mathrm{CDCl}_{3}\right) \delta 213.1,142.5,129.8,78.1,52.7,49.5,46.7$, $40.6,40.2,34.8,26.7,24.8,22.6,22.3,14.8$; Anal. Calcd 
for $\mathrm{C}_{15} \mathrm{H}_{24} \mathrm{O}_{2}: \mathrm{C}, 76.23 ; \mathrm{H}, 10.23$. Found: C, 76.04; H, 10.12. HRMS-EI calcd for $\mathrm{C}_{15} \mathrm{H}_{24} \mathrm{O}_{2}$ [M] $^{+} 236.1776$, found 236.1772 .

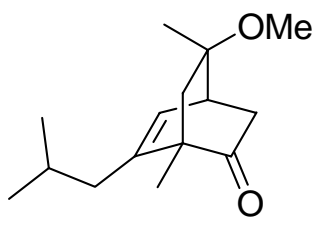

$10 a$

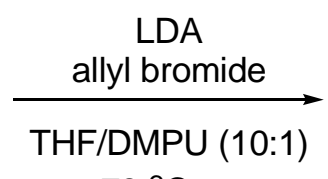

$-78{ }^{\circ} \mathrm{C}$ to $\mathrm{rt}$

$(1 S, 3 S, 4 R, 5 R)$-3-allyl-7-isobutyl-5-methoxy-1, 5-

dimethylbicyclo[2.2.2] oct-7-en-2-one (11a)

To a solution of $i-\mathrm{Pr}_{2} \mathrm{NH}(210 \mu \mathrm{L}, 1.58 \mathrm{mmol})$ in THF (5

$\mathrm{mL})$ and $\mathrm{DMPU}(500 \mu \mathrm{L})$ at $0{ }^{\circ} \mathrm{C}$ was added $n$-BuLi $(1.44 \mathrm{~mL}$, 1.58 mmol, $1.6 \mathrm{M} /$ hexane) dropwise and the reaction was stirred $10 \mathrm{~min}$ at $0{ }^{\circ} \mathrm{C}$. The solution was cooled to $-78{ }^{\circ} \mathrm{C}$ and $10 \mathbf{a}\left(250 \mathrm{mg}, 1.06 \mathrm{mmol}\right.$ ) was added. After 1 h at $-78{ }^{\circ} \mathrm{C}$, freshly distilled allyl bromide (140 $\mu \mathrm{L}, 1.58 \mathrm{mmol})$ was added. The reaction was stirred for 1 h at $-78{ }^{\circ} \mathrm{C}$ and warmed to rt and stirred another $5 \mathrm{~h}$. The reaction was diluted with $\mathrm{Et}_{2} \mathrm{O}$ and a saturated aqueous solution of $\mathrm{NH}_{4} \mathrm{Cl}$ was added. The aqueous layer was extracted with $\mathrm{Et}_{2} \mathrm{O}(3 \times)$, the combined organic layer washed with brine, dried over $\mathrm{Na}_{2} \mathrm{SO}_{4}$, and the solvent evaporated. Purification of the crude by flash chromatography using EtOAc/hexane (10:1) gave 11a

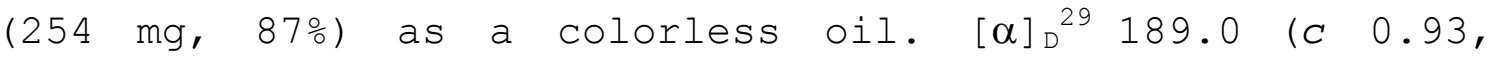


$\left.\mathrm{CHCl}_{3}\right) ; \operatorname{IR}$ (neat) $v=2954,2862,2812,1717,1639,1460$, 1368, 1072, $912 \mathrm{~cm}^{-1} ;{ }^{1} \mathrm{H} \mathrm{NMR}\left(300 \mathrm{MHz}, \mathrm{CDCl}_{3}\right) \delta 5.96(\mathrm{~d}, 1 \mathrm{H}$, $\boldsymbol{J}=6.8 \mathrm{~Hz}), 5.83-5.70(\mathrm{~m}, 1 \mathrm{H}), 5.04-4.98(\mathrm{~m}, 2 \mathrm{H}), 3.12(\mathrm{~s}$, $3 \mathrm{H}), 2.78(\mathrm{dd}, 1 \mathrm{H}, \boldsymbol{J}=6.8,1.7 \mathrm{~Hz}), 2.64-2.48(\mathrm{~m}, 2 \mathrm{H})$, $1.87-1.60(\mathrm{~m}, 5 \mathrm{H}), 1.46(\mathrm{~d}, 1 \mathrm{H}, \boldsymbol{J}=13.8 \mathrm{~Hz}), 1.22(\mathrm{~s}, 3 \mathrm{H})$, $1.12(\mathrm{~s}, 3 \mathrm{H}), 0.86(\mathrm{~d}, 3 \mathrm{H}, \boldsymbol{J}=6.8 \mathrm{~Hz}), 0.84(\mathrm{~d}, 3 \mathrm{H}, \boldsymbol{J}=$ $6.8 \mathrm{~Hz}) ;{ }^{13} \mathrm{C} \operatorname{NMR}\left(75 \mathrm{MHz}, \mathrm{CDCl}_{3}\right) \delta 212.5,141.1,136.6$, 128.4, 116.1, 78.3, 52.9, 49.4, 46.7, 43.5, 42.4, 39.9, $36.1,26.9,24.9,22.8,22.6,15.0$; Anal. Calcd for $\mathrm{C}_{18} \mathrm{H}_{28} \mathrm{O}_{2}$ : C, 78.21; H, 10.21. Found: C, 78.26; H, 10.43. HRMS-EI calcd for $\mathrm{C}_{18} \mathrm{H}_{28} \mathrm{O}_{2}[\mathrm{M}]^{+} 276.2089$, found 276.2084 .

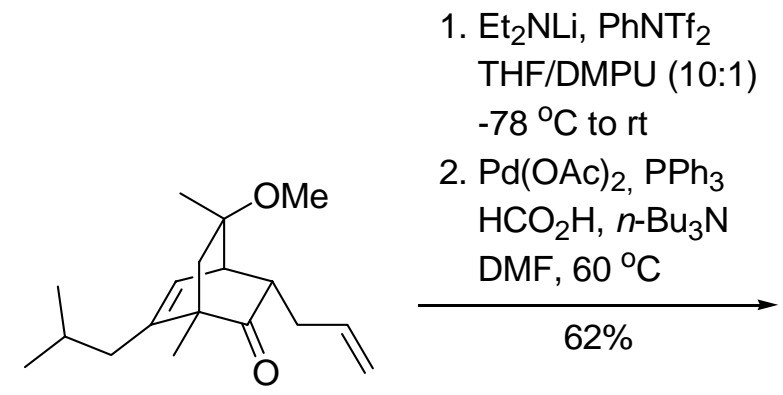

$11 a$

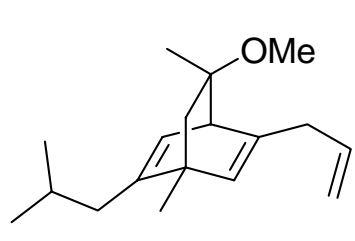

1

\section{$(1 R, 4 R, 8 R)$-5-allyl-2-isobutyl-8-methoxy-1,8-}

\section{dimethylbicyclo[2.2.2] octa-2,5-diene (1)}

To a solution of $\mathrm{Et}_{2} \mathrm{NH}(364 \mu \mathrm{L}, 3.5 \mathrm{mmol})$ in $\mathrm{THF}(3.5$ $\mathrm{mL})$ and $\operatorname{DMPU}(350 \mu \mathrm{L})$ at $0{ }^{\circ} \mathrm{C}$ was added $n$-BuLi $(2.55 \mathrm{~mL}, 3.5$ mmol, $1.4 \mathrm{M} /$ hexane) dropwise and the reaction was stirred 
$10 \mathrm{~min}$ at $0{ }^{\circ} \mathrm{C}$. The solution was cooled to $-78{ }^{\circ} \mathrm{C}$ and $\mathbf{1 1 a}$ $(243 \mathrm{mg}, 0.88 \mathrm{mmol})$ was added. After $1 \mathrm{~h}$ at $-78{ }^{\circ} \mathrm{C}$, a solution of $\operatorname{PhNTf}_{2}(1.25 \mathrm{~g}, 3.5 \mathrm{mmol})$ in $\mathrm{THF}$ (1 mL) was added. The reaction was stirred for 1 h at $-78{ }^{\circ} \mathrm{C}$ and warmed to rt and stirred overnight. The reaction was diluted with $\mathrm{Et}_{2} \mathrm{O}$ and a saturated aqueous solution of $\mathrm{NH}_{4} \mathrm{Cl}$ was added. The aqueous layer was extracted with $\mathrm{Et}_{2} \mathrm{O}(3 \times)$, the combined organic layer washed with brine, dried over $\mathrm{Na}_{2} \mathrm{SO}_{4}$, and the solvent evaporated. The crude product was purified by flash chromatography using Et ${ }_{2} \mathrm{O} /$ pentane $(10: 1)$ to give the triflate contaminated with PhNTf $_{2}$ side-products (348 mg). The crude was used in the next step without further purification. However, an analytically pure sample could be obtained as a colorless oil by additional flash chromatography. $[\alpha]_{D}^{34}-46.2$ ( $\left.0.50, \mathrm{CHCl}_{3}\right) ; \operatorname{IR}$ (neat) $v=$ $2957,1445,1402,1245,1210,1140,1093,1063,1027,862$ $\mathrm{Cm}^{-1} ;{ }^{1} \mathrm{H} \operatorname{NMR}\left(300 \mathrm{MHz}, \mathrm{CDCl}_{3}\right) \delta 5.83(\mathrm{~d}, 1 \mathrm{H}, \boldsymbol{J}=6.3 \mathrm{~Hz})$, $5.78-5.63(\mathrm{~m}, 1 \mathrm{H}), 5.09-4.99(\mathrm{~m}, 2 \mathrm{H}), 3.35(\mathrm{~d}, 1 \mathrm{H}, \mathrm{J}=6.3$ $\mathrm{Hz}), \quad 3.31-3.22(\mathrm{~m}, 1 \mathrm{H}), 3.15(\mathrm{~s}, 3 \mathrm{H}), 2.72(\mathrm{dd}, 1 \mathrm{H}, \mathrm{J}=$ $15.7,7.6 \mathrm{~Hz}), 2.01-1.84(\mathrm{~m}, 2 \mathrm{H}), 1.79-1.68(\mathrm{~m}, 2 \mathrm{H}), 1.41$ $(\mathrm{s}, 3 \mathrm{H}), 1.25(\mathrm{~s}, 3 \mathrm{H}), 0.88(\mathrm{~d}, 3 \mathrm{H}, \mathrm{J}=6.6 \mathrm{~Hz}), 0.86(\mathrm{~d}$, $3 \mathrm{H}, \boldsymbol{J}=6.6 \mathrm{~Hz}) ;{ }^{13} \mathrm{C} \operatorname{NMR}\left(75 \mathrm{MHz}, \mathrm{CDCl}_{3}\right) \delta 149.3,148.4$, $134.3,133.9,126.9,118.5(q, J=320 \mathrm{~Hz}), 117.0,50.7$, 
$50.3,49.7,46.9,39.1,33.9,26.2,24.7,22.8,22.6,15.5$. To a solution of $\mathrm{Pd}(\mathrm{OAC})_{2}(19 \mathrm{mg}, 0.085 \mathrm{mmol}), \mathrm{Ph}_{3} \mathrm{P}$ (45 mg, $0.17 \mathrm{mmol})$, and $n-\mathrm{Bu}_{3} \mathrm{~N}(610 \mu \mathrm{L}, 2.56 \mathrm{mmol})$ in $\mathrm{DMF}$ ( $\left.4 \mathrm{~mL}\right)$ was added the crude triflate $(348 \mathrm{mg})$. The solution was stirred 5 min at room temperature, $\mathrm{HCO}_{2} \mathrm{H}(64 \mu \mathrm{L}, 1.70 \mathrm{mmol})$ was added, and the resulting solution was heated to $60{ }^{\circ} \mathrm{C}$ for 2 h. The reaction mixture was diluted with $\mathrm{Et}_{2} \mathrm{O}$, washed with $2 \mathrm{M}$ aqueous $\mathrm{HCl}(3 \times)$, the combined organic layers washed with brine, dried over $\mathrm{Na}_{2} \mathrm{SO}_{4}$, and the solvent evaporated. Purification of the crude by flash chromatography using Et ${ }_{2} \mathrm{O} /$ pentane (20:1) gave the ligand 1 (142 $\mathrm{mg}, 62 \%$ from 11a) as a colorless liquid). $[\alpha]_{\mathrm{D}}^{33}-83.2$ ( $\left.\mathrm{c} 0.93, \mathrm{CHCl}_{3}\right)$; IR (neat) $v=3037,2955,2823,1638,1461,1366,1120,1072$, $910 \mathrm{~cm}^{-1} ;{ }^{1} \mathrm{H}$ NMR (300 $\left.\mathrm{MHz}, \mathrm{CDCl}_{3}\right) \quad \delta 5.87-5.74(\mathrm{~m}, 2 \mathrm{H})$, $5.67(\mathrm{~d}, 1 \mathrm{H}, \quad J=1.4 \mathrm{~Hz}), 5.04-4.98(\mathrm{~m}, 2 \mathrm{H}), 3.27(\mathrm{dd}, 1 \mathrm{H}$, $J=6.4,1.9 \mathrm{~Hz}), 3.18(\mathrm{~s}, 3 \mathrm{H}), 3.19-2.96(\mathrm{~m}, 1 \mathrm{H}), 2.89-$ $2.81(\mathrm{~m}, 1 \mathrm{H}), 1.91(\mathrm{dd}, 2 \mathrm{H}, \boldsymbol{J}=6.8,1.4 \mathrm{~Hz}) ;{ }^{13} \mathrm{C} \operatorname{NMR}(75$ $\left.\mathrm{MHz}, \mathrm{CDCl}_{3}\right) \delta 149.8, \quad 145.8,136.1,132.1,126.8,115.6$, $83.5,50.7,50.2,50.1,44.9,39.6,39.0,26.6,25.0,22.9$, 22.8, 20.3; Anal. Calcd for $\mathrm{C}_{18} \mathrm{H}_{28} \mathrm{O}: \mathrm{C}, 83.02 ; \mathrm{H}, 10.84$. Found: C, 83.27; $\mathrm{H}$, 11.00. HRMS-EI calcd for $\mathrm{C}_{18} \mathrm{H}_{28} \mathrm{O}$ [M] $^{+}$ 260.2140 , found 260.2138 . 


\section{1,4-addition of Boronic Acids}

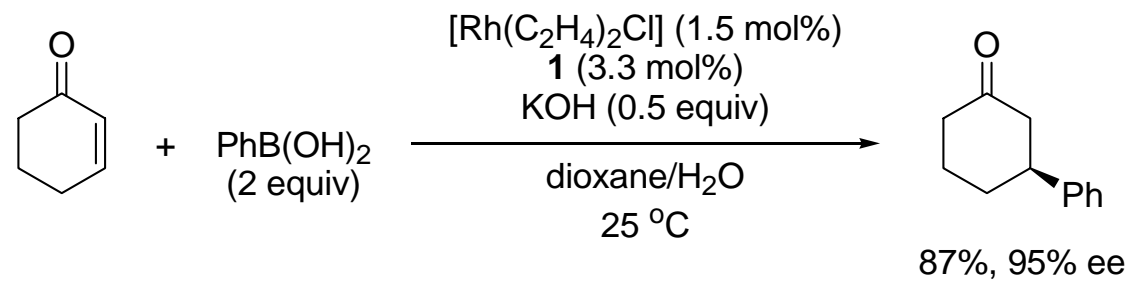

\section{(3S) -3-phenylcyclohexanone. General procedure at $25^{\circ} \mathrm{C}$.}

To $\left[\mathrm{Rh}\left(\mathrm{C}_{2} \mathrm{H}_{4}\right){ }_{2} \mathrm{Cl}\right](2.1 \mathrm{mg}, 5.4 \mu \mathrm{mol})$ and $\mathbf{1}(3.1 \mathrm{mg}, 11.9$ $\mu$ mol) in a Schlenk was added dioxane (1 $\mathrm{mL})$. The resulting solution was stirred for $15 \mathrm{~min}$, and $\mathrm{KOH}\left(1.5 \mathrm{M} / \mathrm{H}_{2} \mathrm{O}, 120 \mu \mathrm{L}\right.$, $0.18 \mathrm{mmol}$ ) was added. After stirring $15 \mathrm{~min}, \mathrm{PhB}(\mathrm{OH})_{2}$ (88 mg, $0.72 \mathrm{mmol}$ ) was added followed by 2-cyclohexenone (35 $\mu \mathrm{L}$, $0.36 \mathrm{mmol}$ ) and the reaction was stirred until completion by TLC. Saturated aqueous $\mathrm{NH}_{4} \mathrm{Cl}$ was added, and the aqueous layer was extracted with $\mathrm{Et}_{2} \mathrm{O}(3 \times)$. The combined organic layers were washed with brine, dried over $\mathrm{MgSO}_{4}$, and the solvent was evaporated to give the crude. The desired product was obtained (55 mg, 87\%) as a colorless liquid after purification by flash chromatography using 20\% Et ${ }_{2} \mathrm{O} /$ hexane. The enantioselectivity was 95\% ee (AD-H, 254 $\mathrm{nm}$, hexane:2-propanol $=98: 2$, flow rate $0.5 \mathrm{ml} / \mathrm{min}) \cdot[\alpha]_{\mathrm{D}}^{27}$ $-18.6\left(c 1.045, \mathrm{CHCl}_{3}\right) \quad\left\{\right.$ lit. $^{1}\left[[\alpha]_{\mathrm{D}}^{20}-22.0\left(\mathrm{C} 1.01, \mathrm{CHCl}_{3}\right)\right\}$. 
All other spectroscopic data was in agreement with the literature. ${ }^{1}$

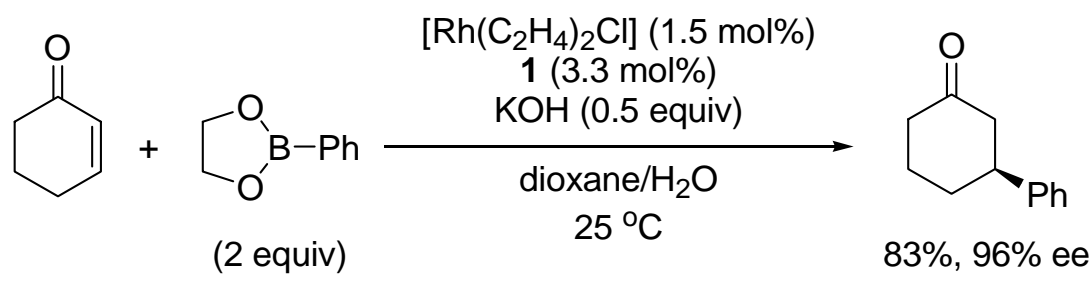

\section{(3S) -3-phenylcyclohexanone .}

Following the general procedure at $25^{\circ} \mathrm{C}$ on a $0.33 \mathrm{mmol}$ scale using 2-cyclohexenone and 2-phenyl-1,3,2dioxaboriane, the desired product (48 mg, 83\%) was isolated by flash chromatography using $20 \% \quad \mathrm{Et}_{2} \mathrm{O} / \mathrm{hexane}$. The enantioselectivity was 96\% ee.

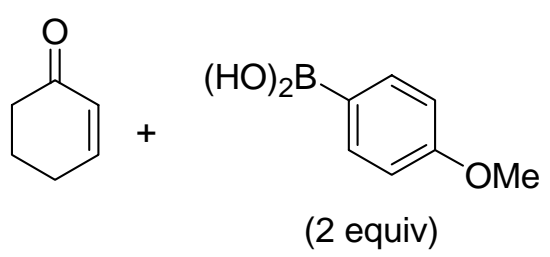

(2 equiv)

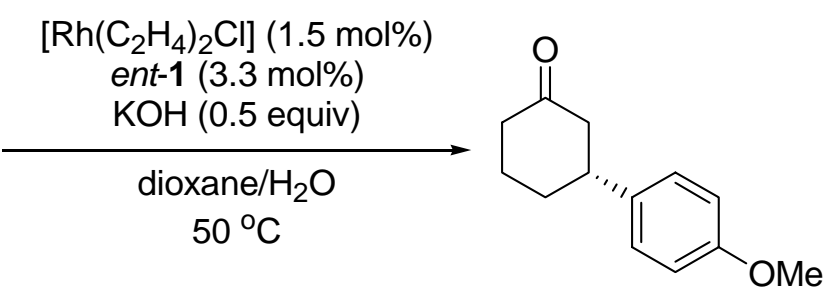

$85 \%, 96 \%$ ee

(3R)-3-(4-methoxyphenyl)cyclohexanone. General procedure at $50{ }^{\circ} \mathrm{C}$.

To $\left[\mathrm{Rh}\left(\mathrm{C}_{2} \mathrm{H}_{4}\right){ }_{2} \mathrm{Cl}\right](1.9 \mathrm{mg}, 4.9 \mu \mathrm{mol})$ and ent-1 $(2.8 \mathrm{mg}$, 10.7 $\mu \mathrm{mol}$ ) in a Schlenk was added dioxane ( $1 \mathrm{~mL})$. The 
resulting solution was stirred for $15 \mathrm{~min}$, and $\mathrm{KOH}$ (1.5 $\mathrm{M} / \mathrm{H}_{2} \mathrm{O}, 113 \mu \mathrm{L}, 0.17 \mathrm{mmol}$ ) was added. After stirring $15 \mathrm{~min}$, 4-MeOPhB $(\mathrm{OH})_{2} \quad(100 \mathrm{mg}, 0.66 \mathrm{mmol})$ was added followed by 2cyclohexenone (32 $\mu \mathrm{L}, 0.33 \mathrm{mmol}$ ) and the reaction was heated at $50{ }^{\circ} \mathrm{C}$ stirred until completion by TLC. Saturated aqueous $\mathrm{NH}_{4} \mathrm{Cl}$ was added, and the aqueous layer was extracted with $\mathrm{Et}_{2} \mathrm{O}(3 \times)$. The combined organic layers were washed with brine, dried over $\mathrm{MgSO}_{4}$, and the solvent was evaporated to give the crude. The desired product was obtained $(57 \mathrm{mg}$, 85\%) was a colorless liquid after purification by flash chromatography using 25\% Et ${ }_{2} \mathrm{O} /$ hexane. The enantioselectivity was 96\% ee (AD-H, $254 \mathrm{~nm}$, hexane:2-propanol = 98:2, flow rate $0.5 \mathrm{ml} / \mathrm{min}) \cdot[\alpha]_{\mathrm{D}}^{33} 13.6\left(\mathrm{c} 1.15, \mathrm{CHCl}_{3}\right) \quad\left\{1 \mathrm{it}{ }^{2}\left[[\alpha]_{\mathrm{D}}{ }^{20}\right.\right.$ 17.2 ( $\left.\left.\mathrm{C} 0.96, \mathrm{CHCl}_{3}\right)\right\}$. All other spectroscopic data was in agreement with the literature. ${ }^{2}$<smiles>COc1ccccc1BOCCO</smiles>

(2 equiv)

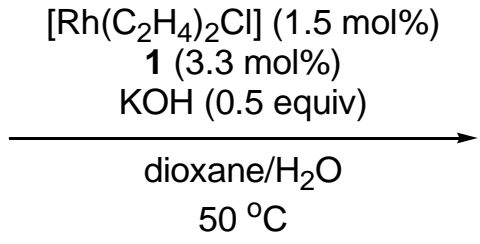

93\%, $94 \%$ ee

\section{(3S) -3-(2-methoxyphenyl)cyclohexanone}

Following the general procedure at $50{ }^{\circ} \mathrm{C}$ on a $0.34 \mathrm{mmol}$ scale using 2-cyclohexenone and 2-methoxylphenylboronic 
acid, the desired product (69 mg, 93\%) was isolated by flash chromatography using 20\% Et ${ }_{2} \mathrm{O} / \mathrm{hexane}$. The enantioselectivity was 94\% ee (OD, $254 \mathrm{~nm}$, hexane:2propanol $=95: 5$, flow rate $1.0 \mathrm{ml} / \mathrm{min}) \cdot[\alpha]_{\mathrm{D}}^{32}-36.3$ (c 1.02, $\left.\mathrm{CHCl}_{3}\right)$. All other spectroscopic data was in agreement with the literature. ${ }^{3}$

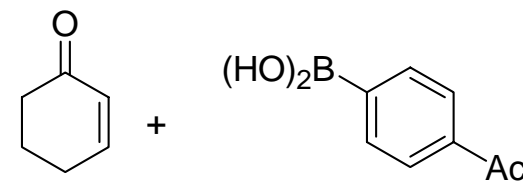

(2 equiv)

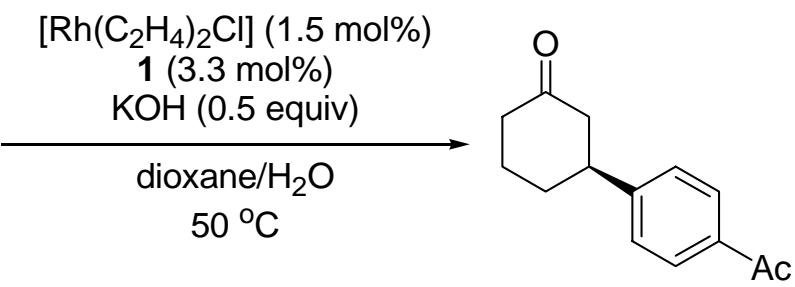

$96 \%, 97 \%$ ee

\section{(3S) -3-(4-acetylphenyl)cyclohexanone}

Following the general procedure at $50{ }^{\circ} \mathrm{C}$ on a $0.38 \mathrm{mmol}$ scale using 2-cyclohexenone and 4-acetylphenylboronic acid, the desired product $(79 \mathrm{mg}, 96 \%)$ was isolated by flash chromatography using $30 \%$ EtOAc/hexane. The enantioselectivity was 97\% ee (AD-H, $254 \mathrm{~nm}$, hexane:2propanol $=90: 10$, flow rate $0.5 \mathrm{ml} / \mathrm{min}) \cdot[\alpha]_{\mathrm{D}}^{32}-7.8$ (c 1.02, $\left.\mathrm{CHCl}_{3}\right) ; \mathrm{IR}$ (neat) $v=2940,1737,1717,1667,1365,1268$, 1226, 1217, 960, $827 \mathrm{~cm}^{-1} ;{ }^{1} \mathrm{H} \mathrm{NMR}\left(300 \mathrm{MHz}, \mathrm{CDCl}_{3}\right) \delta 7.87(\mathrm{~m}$, $2 \mathrm{H}), 7.26(\mathrm{~m}, 2 \mathrm{H}), 3.02(\mathrm{~m}, 1 \mathrm{H}), 2.59-2.28(\mathrm{~m}, 7 \mathrm{H}), 2.15-$ $2.01(\mathrm{~m}, 2 \mathrm{H}), 1.86-1.70(\mathrm{~m}, 2 \mathrm{H}) ;{ }^{13} \mathrm{C} \mathrm{NMR}\left(75 \mathrm{MHz}, \mathrm{CDCl}_{3}\right) \delta$ 
$210.0,197.4,149.5,135.6,128.7,126.7,48.4,44.6,41.1$, $32.5,26.6,25.5 ; \operatorname{MS}-\operatorname{ESI}(\boldsymbol{m} / \mathbf{z}) 239[\mathrm{M}+\mathrm{Na}]^{+}$.<smiles>O=C1C=[C+]CCC1</smiles>

(2 equiv)
$\left[\mathrm{Rh}\left(\mathrm{C}_{2} \mathrm{H}_{4}\right)_{2} \mathrm{Cl}\right](1.5 \mathrm{~mol} \%)$

$1(3.3 \mathrm{~mol} \%)$

$\mathrm{KOH}$ (0.5 equiv)

$50{ }^{\circ} \mathrm{C}$

$94 \%, 97 \%$ ee

\section{(3S) -3-(2-fluorophenyl)cyclohexanone}

Following the general procedure at $50{ }^{\circ} \mathrm{C}$ on a $0.36 \mathrm{mmol}$ scale using 2-cyclohexenone and 2-fluorophenylboronic acid, the desired product (69 $\mathrm{mg}, 93 \%)$ was isolated by flash chromatography using $20 \% \mathrm{Et}_{2} \mathrm{O} / \mathrm{hexane}$. The enantioselectivity was 97\% ee (OD, $254 \mathrm{~nm}$, hexane:2-propanol = 99.5:0.5, flow rate $1.0 \mathrm{ml} / \mathrm{min}) \cdot[\alpha]_{\mathrm{D}}^{32}-12.2$ (C $\left.1.40, \mathrm{CHCl}_{3}\right)$. All other spectroscopic data was in agreement with the literature. ${ }^{3}$

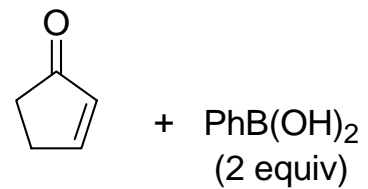

(2 equiv)

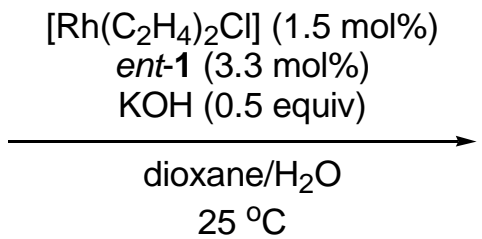

$25{ }^{\circ} \mathrm{C}$

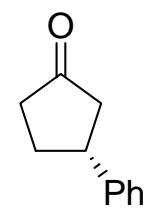

91\%, 94\% ee

\section{(3R) - 3-phenylcyclopentanone}

Following the general procedure at $25^{\circ} \mathrm{C}$ on a $0.30 \mathrm{mmol}$ scale using 2-cyclopentenone and phenylboronic acid, the desired product (44 mg, 91\%) was isolated by flash 
chromatography using EtOAc/hexane $(5: 1)$. The enantioselectivity was 94\% ee (OBH, $254 \mathrm{~nm}$, hexane:2propanol $=99: 1$, flow rate $1.0 \mathrm{ml} / \mathrm{min}) \cdot[\alpha]_{D}^{33} 24.2$ (c 0.49, $\left.\mathrm{CHCl}_{3}\right) . \quad\left\{\right.$ lit. $^{4}\left[[\alpha]_{\mathrm{D}}{ }^{20} 42.0 \quad\left(\mathrm{C} 1.07, \mathrm{CHCl}_{3}\right)\right\}$. All other spectroscopic data was in agreement with the literature. ${ }^{4}$

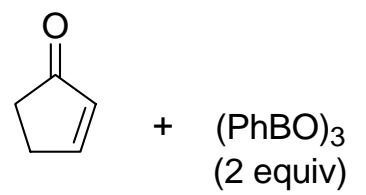

$\left[\mathrm{Rh}\left(\mathrm{C}_{2} \mathrm{H}_{4}\right)_{2} \mathrm{Cl}\right](1.5 \mathrm{~mol} \%)$

1 (3.3 mol\%)

$\mathrm{KOH}(0.5$ equiv)

dioxane/ $\mathrm{H}_{2} \mathrm{O}$

$25{ }^{\circ} \mathrm{C}$

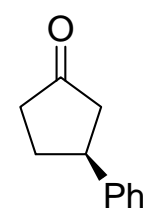

$95 \%, 97 \%$ ee

\section{(3S) -3-phenylcyclopentanone}

Following the general procedure at $25{ }^{\circ} \mathrm{C}$ on a $0.30 \mathrm{mmol}$ scale using 2-cyclopentenone and phenylboric anhydride, the desired product (46 mg, 95\%) was isolated by flash chromatography using EtOAc/hexane $(5: 1)$. The enantioselectivity was $97 \%$ ee.

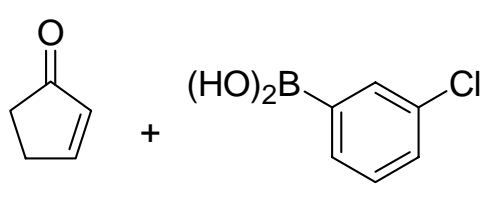

(2 equiv)

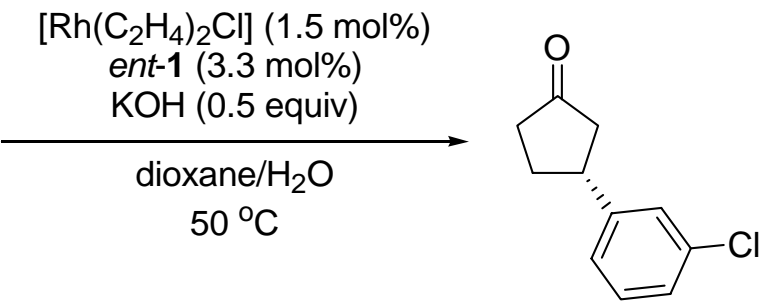

$98 \%, 95 \%$ ee

\section{(3R) -3-(3-chlorophenyl)cyclopentanone}

Following the general procedure at $50{ }^{\circ} \mathrm{C}$ on a $0.34 \mathrm{mmol}$ scale using 2-cyclopentenone and 3-chlorophenylboronic 
acid, the desired product (65 $\mathrm{mg}$, 98\%) was isolated by flash chromatography using 20\% Et ${ }_{2} \mathrm{O} /$ hexane. The enantioselectivity was 95\% ee (OB-H, $254 \mathrm{~nm}$, hexane:2propanol $=98: 2$, flow rate $1.0 \mathrm{ml} / \mathrm{min}) \cdot[\alpha]_{D}^{33} 57.9$ ( C 0.95, $\left.\mathrm{CHCl}_{3}\right) . \quad\left\{\right.$ lit. $^{2}\left[[\alpha]_{\mathrm{D}}{ }^{20} 75.3\right.$ (C $\left.\left.0.96, \mathrm{CHCl}_{3}\right)\right\}$. All other spectroscopic data was in agreement with the literature. ${ }^{2}$

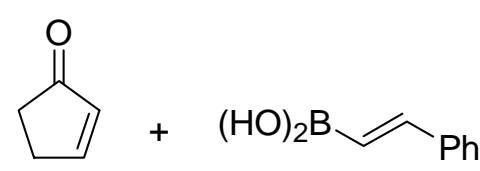

(2 equiv)

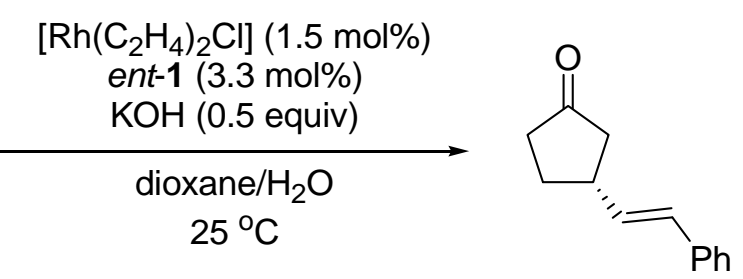

97\%, 90\% ee

\section{$(3 R)-3-[(E)-2$-phenylvinyl] cyclopentanone}

Following the general procedure at $25{ }^{\circ} \mathrm{C}$ on a $0.30 \mathrm{mmol}$ scale using 2-cyclopentenone and trans-2phenylvinylphenylboronic acid, the desired product (53 mg, 97\%) was isolated by flash chromatography using EtOAc/hexane (4:1). The enantioselectivity was 90\% ee (ADH, $254 \mathrm{~nm}$, hexane:2-propanol = 99:1, flow rate $1.0 \mathrm{ml} / \mathrm{min})$. $[\alpha]_{D}^{33} 70.7$ ( $\left.\mathrm{C} 0.49, \mathrm{CHCl}_{3}\right)$. All other spectroscopic data was in agreement with the literature. ${ }^{5}$ 


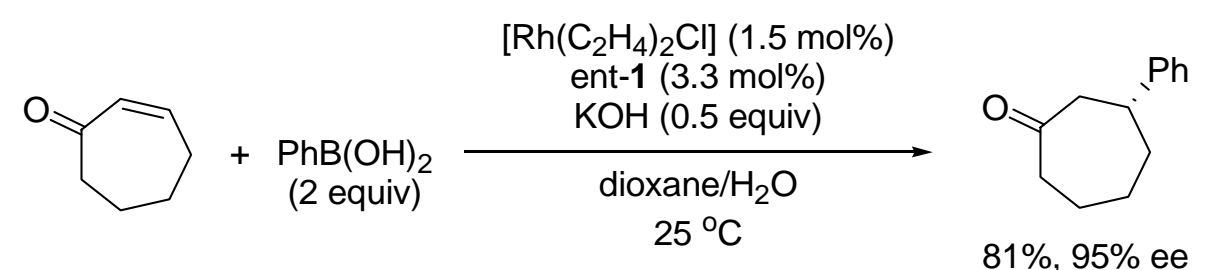

\section{(3R) -3-phenylcycloheptanone}

Following the general procedure at $25^{\circ} \mathrm{C}$ on a $0.30 \mathrm{mmol}$ scale using 2-cycloheptenone and phenylboronic acid, the desired product (49 mg, 81\%) was isolated by flash chromatography using EtOAc/hexane (5:1). The enantioselectivity was 95\% ee (AD-H, 254 nm, hexane:2propanol $=98: 2$, flow rate $0.5 \mathrm{ml} / \mathrm{min}) \cdot[\alpha]_{\mathrm{D}}^{33} 116.8$ (c 0.35 , $\left.\mathrm{CHCl}_{3}\right)$. All other spectroscopic data was in agreement with the literature. ${ }^{6}$
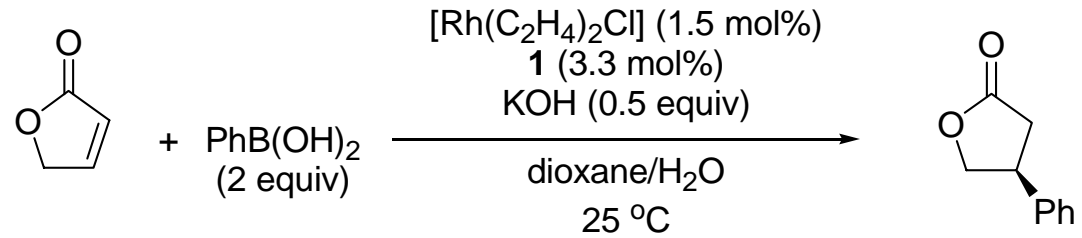

$80 \%, 90 \%$ ee

\section{(4S) - 4-phenyldihydro-2(3H) - furanone}

Following the general procedure at $25^{\circ} \mathrm{C}$ on a $0.36 \mathrm{mmol}$ scale using 2-(5H)-furanone and phenylboronic acid, the desired product (39 $\mathrm{mg}, 80 \%)$ was isolated by flash chromatography using EtOAc/hexane (4:1). The enantioselectivity was 90\% ee (AD-H, 254 nm, hexane:2propanol $=98: 2$, flow rate $1.0 \mathrm{ml} / \mathrm{min}) \cdot[\alpha]_{\mathrm{D}}^{33} 45.8$ (c 0.76 , 
$\left.\mathrm{CHCl}_{3}\right) \cdot \quad\left\{\right.$ lit. $^{7}\left[[\alpha]_{\mathrm{D}}^{20} 49.0 \quad\left(\mathrm{C} 3.1, \mathrm{CHCl}_{3}\right)\right\}$. All other spectroscopic data was in agreement with the literature. ${ }^{7}$

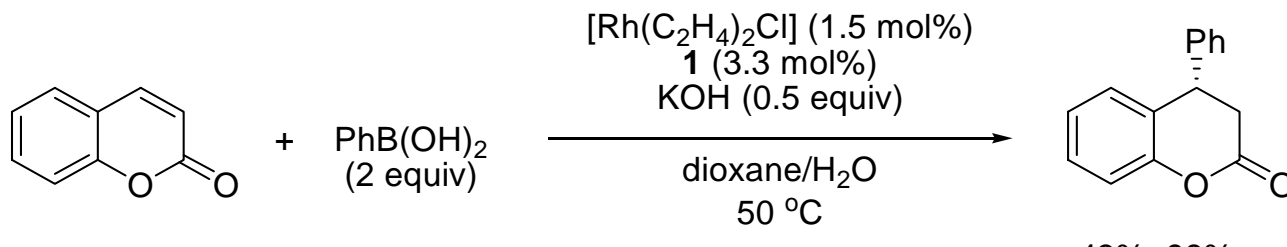

$43 \%, 98 \%$ ee

\section{(4S) -4-phenyl-2-chromanone}

Following the general procedure at $50{ }^{\circ} \mathrm{C}$ on a $0.30 \mathrm{mmol}$ scale using coumarin and phenylboronic acid, the desired product (29 mg, 43\%) was isolated by flash chromatography using EtOAc/hexane $(7: 1)$. The enantioselectivity was 98\% ee (OJ-H, $254 \mathrm{~nm}$, hexane:2-propanol = 97:3, flow rate 1.0

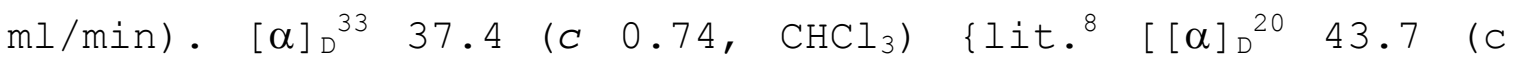
$\left.\left.5.58, \mathrm{C}_{6} \mathrm{H}_{6}\right)\right\}$. All other spectroscopic data was in agreement with the literature. ${ }^{9}$

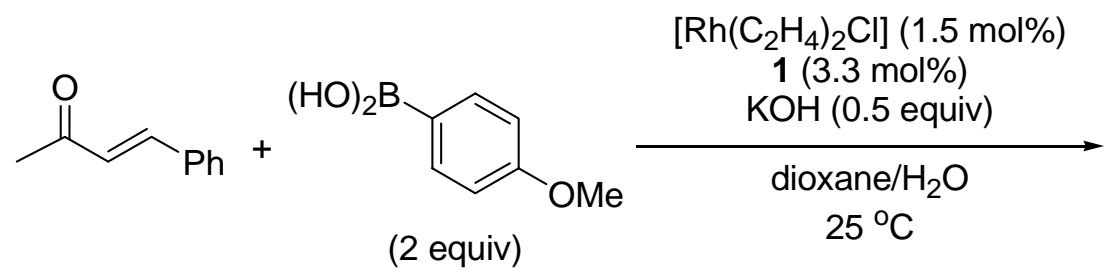

(4S) -4- (4-methoxyphenyl) -4-phenyl-2-butanone<smiles>COc1ccc([C@H](CC(C)=O)c2ccccc2)cc1</smiles>

$68 \%, 90 \%$ ee

Following the general procedure at $25{ }^{\circ} \mathrm{C}$ on a $0.30 \mathrm{mmol}$ scale using trans-4-phenyl-3-buten-2-one and 4- 
methoxyphenylboronic acid, the desired product (52 mg, 68\%) was isolated by flash chromatography using EtoAc/hexane (5:1). The enantioselectivity was 90\% ee (OD-H, $254 \mathrm{~nm}$, hexane:2-propanol $=98: 2$, flow rate $1.0 \mathrm{ml} / \mathrm{min}) \cdot[\alpha]_{\mathrm{D}}^{33}-0.6$ (c $\left.0.72, \mathrm{CHCl}_{3}\right)$. All other spectroscopic data was in agreement with the literature. ${ }^{10}$

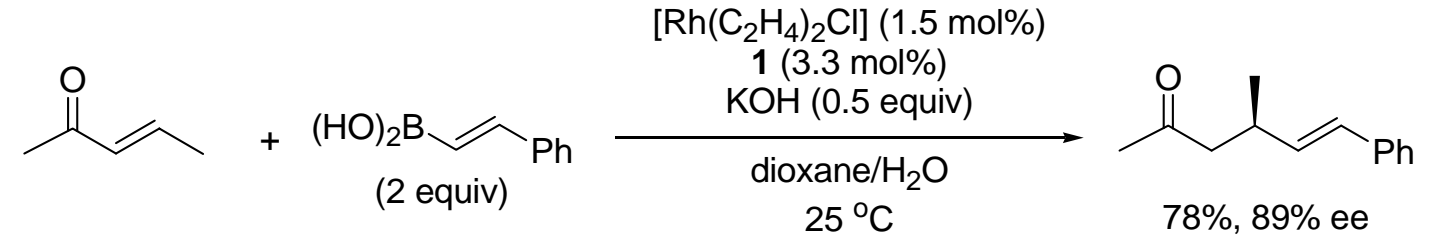

\section{(4R, 5E) - 4-methyl-6-phenyl-5-hexen-2-one}

Following the general procedure at $25{ }^{\circ} \mathrm{C}$ on a $0.26 \mathrm{mmol}$ scale using 3-penten-2-one and trans-2-phenylvinylboronic acid, the desired product $(37 \mathrm{mg}, 78 \%$ was isolated by flash chromatography using EtOAc/hexane (5:1). The enantioselectivity was 89\% ee (AD-H, $254 \mathrm{~nm}$, hexane:2propanol $=99: 1$, flow rate $0.8 \mathrm{ml} / \mathrm{min}) \cdot[\alpha]_{\mathrm{D}}^{24}-58.4$ (c 0.85 , $\left.\mathrm{CHCl}_{3}\right) \cdot\left\{\right.$ lit. $^{11}\left[[\alpha]_{\mathrm{D}}^{20}-57.3\left(\mathrm{C} 0.78, \mathrm{CHCl}_{3}\right)\right\}$. All other spectroscopic data was in agreement with the literature. ${ }^{11}$ 


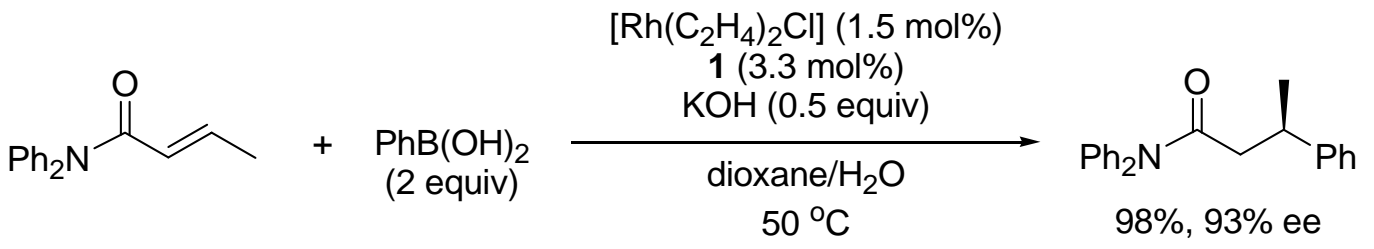

\section{(3R) - N, N, 3-triphenylbutanamide}

Following the general procedure at $50{ }^{\circ} \mathrm{C}$ on a $0.30 \mathrm{mmol}$

scale using (2E)-N,N-diphenyl-2-butenamide and phenylboronic acid, the desired product (93 mg, 93\%) was isolated by flash chromatography using EtOAc/hexane (7:1). The enantioselectivity was 93\% ee (OD-H, 254 nm, hexane:2propanol $=98: 2$, flow rate $1.0 \mathrm{ml} / \mathrm{min}) \cdot[\alpha]_{\mathrm{D}}^{32}-71.4$ (c 0.64 , $\mathrm{CHCl}_{3}$ ); IR (neat) $v=3060,3027,2962,1666,1591,1489$, 1451, 1363, $1292 \mathrm{~cm}^{-1} ;{ }^{1} \mathrm{H} \operatorname{NMR}\left(300 \mathrm{MHz}, \mathrm{CDCl}_{3}\right) \delta 7.31-7.01$ $(\mathrm{m}, 15 \mathrm{H}), 3.40-3.18(\mathrm{~m}, 1 \mathrm{H}), 2.44-2.61(\mathrm{~m}, 2 \mathrm{H}), 1.29(\mathrm{~d}$, $3 \mathrm{H}, \quad \boldsymbol{J}=7.2 \mathrm{~Hz}) ;{ }^{13} \mathrm{C} \operatorname{NMR}\left(75 \mathrm{MHz}, \mathrm{CDCl}_{3}\right) \delta 171.7, \quad 145.7$, $142.7,128.3,127.1,126.2,43.6,37.3,21.6$.

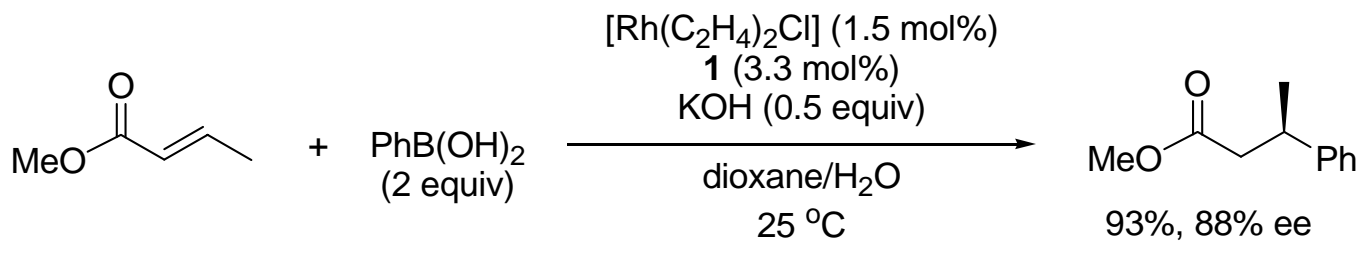

\section{(3R)-Methyl 3-phenylbutanoate}

Following the general procedure at $25{ }^{\circ} \mathrm{C}$ on a $0.30 \mathrm{mmol}$ scale using methyl crotonate and phenylboronic acid, the desired product (50 mg, 93\%) was isolated by flash 
chromatography using EtOAc/hexane (10:1). The enantioselectivity was 88\% ee (OB-H, 254 nm, hexane:2propanol $=99: 1$, flow rate $1.0 \mathrm{ml} / \mathrm{min}) \cdot[\alpha]_{\mathrm{D}}^{33}-18.9$ (c 0.72 , $\left.\mathrm{CHCl}_{3}\right) \cdot\left\{\right.$ lit. $^{12}\left[[\alpha]_{\mathrm{D}}^{22}-29.4\left(\mathrm{C} 1.02, \mathrm{CHCl}_{3}\right)\right\}$. All other spectroscopic data was in agreement with the literature.

\section{Characterization data for the other ligands prepared}

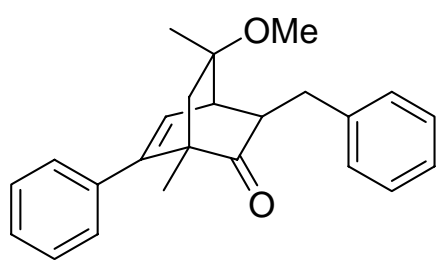

$(1 S, 3 S, 4 R, 5 R)$-3-benzyl-5-methoxy-1, 5-dimethyl-7phenylbicyclo[2.2.2] oct-7-en-2-one $[\alpha]_{\mathrm{D}}^{29}-2.7\left(\mathrm{C} 0.53, \mathrm{CHCl}_{3}\right) ; \mathrm{IR}$ (neat) $v=3026,2970,2933$, 2826, 1715, 1452, 1085, 1071, 1055, 762, $744 \mathrm{~cm}^{-1} ;{ }^{1} \mathrm{H}$ NMR $\left(300 \mathrm{MHz}, \mathrm{CDCl}_{3}\right) \delta 7.19-7.32(\mathrm{~m}, 8 \mathrm{H}), 7.03-7.06(\mathrm{~m}, 2 \mathrm{H})$, $6.23(\mathrm{~d}, 1 \mathrm{H}, J=6.9 \mathrm{~Hz}), 3.34-3.38(\mathrm{~m}, 1 \mathrm{H}), 2.91(\mathrm{~s}, 3 \mathrm{H})$, $2.87(\mathrm{~m}, 1 \mathrm{H}), 2.81(\mathrm{dd}, 1 \mathrm{H}, \boldsymbol{J}=6.9 \mathrm{~Hz}, \boldsymbol{J}=1.9 \mathrm{~Hz}), 2.30-$ $2.39(\mathrm{~m}, 1 \mathrm{H}), 1.90(\mathrm{~d}, 1 \mathrm{H}, J=14.0 \mathrm{~Hz}), 1.70(\mathrm{~d}, 1 \mathrm{H}, J=$ 13.7 Hz), $1.27(\mathrm{~s}, 3 \mathrm{H}), 1.08(\mathrm{~s}, 3 \mathrm{H}) ;{ }^{13} \mathrm{C}$ NMR $(75 \mathrm{MHz}$, $\left.\mathrm{CDCl}_{3}\right) \delta 211.7,144.2,139.9,138.1,131.8,128.7,128.4$, 128.2, 127.9, 127.3, 126.2, 78.7, 53.0, 49.2, 47.7, 44.8, 
43.2, 37.6, 25.1, 16.7; HRMS-EI calcd for $\mathrm{C}_{24} \mathrm{H}_{26} \mathrm{O}_{2}$ [M] $^{+}$ 346.1933, found 346.1927.

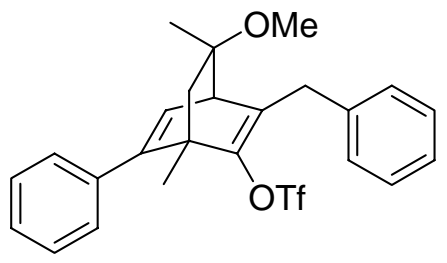

$(1 S, 4 R, 8 R)$ - 3 - benzyl - 8 - methoxy -1, 8-dimethyl-6 -

phenylbicyclo[2.2.2]octa-2,5-dien-2yltrifluromethanesulfonate

$[\alpha]_{\mathrm{D}}^{34}-174.5$ (c $\left.0.69, \mathrm{CHCl}_{3}\right) ; \operatorname{IR}$ (neat) $v=2968,2938$, $2824,1686,1599,1400,1207,1136,1086,1063,1046,865$, $764 \mathrm{~cm}^{-1} ;{ }^{1} \mathrm{H} \operatorname{NMR}\left(300 \mathrm{MHz}, \mathrm{CDCl}_{3}\right) \delta 7.21-7.34(\mathrm{~m}, 6 \mathrm{H}), 7.12-$ $7.15(\mathrm{~m}, 4 \mathrm{H}), 6.00(\mathrm{~d}, 1 \mathrm{H}, \boldsymbol{J}=6.5 \mathrm{~Hz}), 4.12(\mathrm{~d}, 1 \mathrm{H}, \boldsymbol{J}=$ $15.6 \mathrm{~Hz}), 3.32(\mathrm{~d}, 1 \mathrm{H}, \boldsymbol{J}=16.5 \mathrm{~Hz}), 3.31(\mathrm{~d}, 1 \mathrm{H}, \boldsymbol{J}=6.2$ $\mathrm{Hz}), 3.20(\mathrm{~s}, 3 \mathrm{H}), 1.95(\mathrm{~d}, 1 \mathrm{H}, \boldsymbol{J}=12.5 \mathrm{~Hz}), 1.48(\mathrm{~d}, 1 \mathrm{H}$, $\boldsymbol{J}=12.1 \mathrm{~Hz}), 1.36(\mathrm{~s}, 3 \mathrm{H}), 1.31(\mathrm{~s}, 3 \mathrm{H}) ;{ }^{13} \mathrm{C} \mathrm{NMR}(75 \mathrm{MHz}$, $\left.\mathrm{CDCl}_{3}\right) \delta 185.2,150.9,140.6,137.5,135.2,130.9,129.0$, $128.4,128.3,127.9,127.2,126.3,83.3,51.0,50.7,50.5$, $47.3,35.8,24.8,17.7$; Anal. Calcd for $\mathrm{C}_{25} \mathrm{H}_{25} \mathrm{~F}_{3} \mathrm{O}_{4} \mathrm{~S}: \mathrm{C}$, 62.75; H, 5.27. Found: C, 63.02; H, 5.27 . 


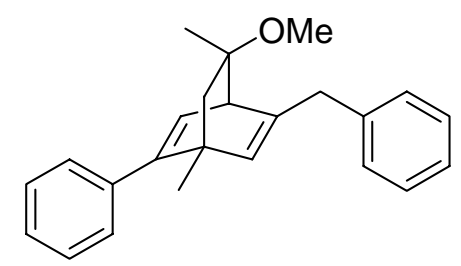

$(1 R, 4 R, 8 R)$-5 - benzyl-8-methoxy-1,8-dimethyl-2-

phenylbicyclo[2.2.2] octa-2,5-diene (2)

$\left.[\alpha]_{D}^{33}-150.4(c) 0.73, \mathrm{CHCl}_{3}\right) ; \operatorname{IR}$ (neat) $v=3020,2961$, 2823, 1601, 1493, 1452, 1366, 1213, 1117, 1071, $751 \mathrm{~cm}^{-1} ;{ }^{1} \mathrm{H}$ $\operatorname{NMR}\left(300 \mathrm{MHz}, \mathrm{CDCl}_{3}\right) \delta 7.06-7.33(\mathrm{~m}, 10 \mathrm{H}), 6.01(\mathrm{~d}, 1 \mathrm{H}, J=$ $6.2 \mathrm{~Hz}), 5.79(\mathrm{~s}, 1 \mathrm{H}), 3.72(\mathrm{~d}, 1 \mathrm{H}, J=15.9 \mathrm{~Hz}), 3.47(\mathrm{~d}$, $1 \mathrm{H}, J=15.9 \mathrm{~Hz}), 3.35(\mathrm{dd}, 1 \mathrm{H}, J=6.5 \mathrm{~Hz}, J=1.9 \mathrm{~Hz})$ ， $3.24(\mathrm{~s}, 3 \mathrm{H}), 1.55(\mathrm{~d}, 1 \mathrm{H}, J=11.8 \mathrm{~Hz}), 1.44(\mathrm{~d}, 1 \mathrm{H}, J=$ $11.8 \mathrm{~Hz}), 1.31(\mathrm{~s}, 3 \mathrm{H}), 1.29(\mathrm{~s}, 3 \mathrm{H}) ;{ }^{13} \mathrm{C} \mathrm{NMR}(75 \mathrm{MHz}$, $\left.\mathrm{CDCl}_{3}\right) \delta 151.7,146.8,139.4,133.2,131.4,129.1,128.1$, $127.9,127.6,126.5,125.8,84.0,51.1,50.8,50.3,45.1$,

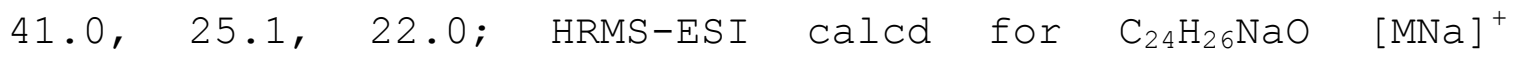
353.1876, found 353.1881. 


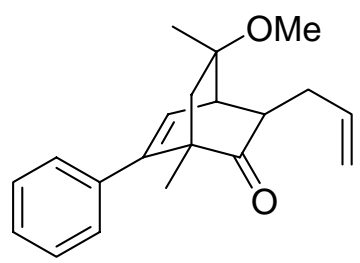

$(1 S, 3 S, 4 R, 5 R)$ - 3-allyl-5-methoxy-1, 5-dimethyl-7-

phenylbicyclo[2.2.2] oct-7-en-2-one

$[\alpha]_{\mathrm{D}}^{31} 115.3\left(\mathrm{c} 1.74, \mathrm{CHCl}_{3}\right) ; \mathrm{IR}$ (neat) $v=3016,2970,2943$, 2933, 1737, 1442, 1366, 1228, 1216, 1075, 912, $699 \mathrm{~cm}^{-1} ;{ }^{1} \mathrm{H}$ $\operatorname{NMR}\left(300 \mathrm{MHz}, \mathrm{CDCl}_{3}\right) \delta 7.46-7.54(\mathrm{~m}, 3 \mathrm{H}), 7.23-7.26(\mathrm{~m}, 2 \mathrm{H})$, $6.44(\mathrm{~d}, 1 \mathrm{H}, \boldsymbol{J}=6.9 \mathrm{~Hz}), 5.95-6.08(\mathrm{~m}, 1 \mathrm{H}), 5.23-5.29(\mathrm{~m}$, $2 \mathrm{H}), 3.41(\mathrm{~s}, 3 \mathrm{H}), 3.17(\mathrm{~d}, 1 \mathrm{H}, \boldsymbol{J}=6.9 \mathrm{~Hz}), 2.86-2.92(\mathrm{~m}$, $2 \mathrm{H}), 2.07-2.18(\mathrm{~m}, 1 \mathrm{H}), 2.12(\mathrm{~d}, 1 \mathrm{H}, \boldsymbol{J}=14.0 \mathrm{~Hz}), 1.89(\mathrm{~d}$, $1 \mathrm{H}, \quad \boldsymbol{J}=14.0 \mathrm{~Hz}), 1.56(\mathrm{~s}, 3 \mathrm{H}), 1.25(\mathrm{~s}, 3 \mathrm{H}) ;{ }^{13} \mathrm{C} \mathrm{NMR}(75$ $\left.\mathrm{MHz}, \mathrm{CDCl}_{3}\right) \delta 212.0,143.9,138.1,136.4,132.0,128.1$, $127.8,127.2,116.5,78.6,53.0,49.6,47.2,44.1,42.5$, 36.2, 25.2, 16.7; Anal. Calcd for $\mathrm{C}_{20} \mathrm{H}_{24} \mathrm{O}_{2}: \mathrm{C}, 81.04 ; \mathrm{H}$, 8.16. Found: C, 81.15; $\mathrm{H}, 8.30$; HRMS-EI calcd for $\mathrm{C}_{20} \mathrm{H}_{24} \mathrm{O}_{2}$ $[\mathrm{M}]^{+} 296.1776$, found 296.1772 . 


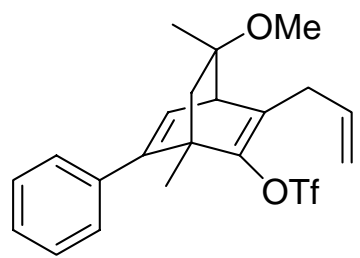

$(1 S, 4 R, 8 R)$-3-allyl-8-methoxy-1, 8-dimethyl-6-

phenylbicyclo[2.2.2] octa-2,5-dien-2yltrifluromethanesulfonate

$[\alpha]_{\mathrm{D}}^{34}-126.3\left(\mathrm{C} 1.42, \mathrm{CHCl}_{3}\right) ;$ IR (neat) $v=2976,2828$, 1686, 1639, 1400, 1206, 1137, 1088, 1046, $860 \mathrm{~cm}^{-1} ;{ }^{1} \mathrm{H}$ NMR (300 MHz, $\left.\mathrm{CDCl}_{3}\right) \delta 7.25-7.35(\mathrm{~m}, 3 \mathrm{H}), 7.10-7.13(\mathrm{~m}, 2 \mathrm{H})$, $6.12(\mathrm{~d}, 1 \mathrm{H}, J=6.5 \mathrm{~Hz}), 5.71-5.85(\mathrm{~m}, 1 \mathrm{H}), 5.06-5.13(\mathrm{~m}$, $2 \mathrm{H}), 3.53(\mathrm{~d}, 1 \mathrm{H}, J=6.2 \mathrm{~Hz}), 3.32-3.48(\mathrm{~m}, 1 \mathrm{H}), 3.22(\mathrm{~s}$, $3 \mathrm{H}), 2.78-2.85(\mathrm{~m}, 1 \mathrm{H}), 1.90(\mathrm{~d}, 1 \mathrm{H}, J=12.4 \mathrm{~Hz}), 1.47(\mathrm{~d}$, $1 \mathrm{H}, J=12.1 \mathrm{~Hz}), 1.39(\mathrm{~s}, 3 \mathrm{H}), 1.31(\mathrm{~s}, 3 \mathrm{H}) ;{ }^{13} \mathrm{C} \operatorname{NMR}(75$ $\left.\mathrm{MHz}, \mathrm{CDCl}_{3}\right) \delta 151.3,148.1,137.5,134.6,133.7,130.6$, 128.2, 127.8, 127.1, 117.1, 83.3, 51.2, 50.5, 50.4, 47.3, 34.2, 24.9, 17.4; Anal. Calcd for $\mathrm{C}_{21} \mathrm{H}_{23} \mathrm{~F}_{3} \mathrm{O}_{4} \mathrm{~S}: \mathrm{C}, 58.87 ; \mathrm{H}$, 5.41. Found: C, 58.68; H, 5.63. HRMS-ESI calcd for $\mathrm{C}_{21} \mathrm{H}_{23} \mathrm{~F}_{3} \mathrm{NaO}_{4} \mathrm{~S}$ [MNa] ${ }^{+}$451.1161, found 451.1163. 


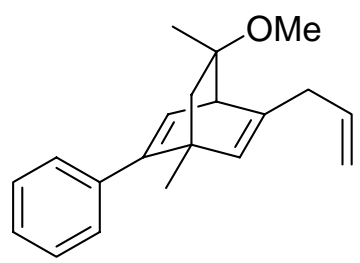

$(1 R, 4 R, 8 R)$-5-allyl-8-methoxy-1, 8-diemthyl-2-

phenylbicyclo[2.2.2] octa-2,5-diene (3)

$\left.[\alpha]_{D}{ }^{33}-150.3(C) 0.84, \mathrm{CHCl}_{3}\right) ; \operatorname{IR}$ (neat) $v=3040,2961$, 2822, 1637, 1597, 1454, 1366, 1119, 1071, 910, $756 \mathrm{~cm}^{-1} ;{ }^{1} \mathrm{H}$ $\operatorname{NMR}\left(300 \mathrm{MHz}, \mathrm{CDCl}_{3}\right) \delta 7.21-7.32(\mathrm{~m}, 3 \mathrm{H}), 7.06-7.09(\mathrm{~m}, 2 \mathrm{H})$, $6.09(\mathrm{~d}, 1 \mathrm{H}, J=6.5 \mathrm{~Hz}), 5.79-5.93(\mathrm{~m}, 2 \mathrm{H}), 5.03-5.10(\mathrm{~m}$, $2 \mathrm{H}), 3.43(\mathrm{dd}, 1 \mathrm{H}, J=6.5 \mathrm{~Hz}, \boldsymbol{J}=1.9 \mathrm{~Hz}), 3.23(\mathrm{~s}, 3 \mathrm{H})$, $2.88-3.23(\mathrm{~m}, 2 \mathrm{H}), 1.53(\mathrm{~d}, 1 \mathrm{H}, J=12.1 \mathrm{~Hz}), 1.43(\mathrm{~d}, 1 \mathrm{H}$, $J=11.8 \mathrm{~Hz}), 1.34(\mathrm{~s}, 3 \mathrm{H}), 1.29(\mathrm{~s}, 3 \mathrm{H}) ;{ }^{13} \mathrm{C} \mathrm{NMR}(75 \mathrm{MHz}$ ， $\left.\mathrm{CDCl}_{3}\right) \delta 152.0,146.1,139.4,135.9,132.3,131.1,127.8$, 127.6, 126.4, 115.9, 83.9, 51.2, 50.7, 50.3, 45.1, 39.1, 25.1, 22.0; Anal. Calcd for $\mathrm{C}_{20} \mathrm{H}_{24} \mathrm{O}: \mathrm{C}, 85.67 ; \mathrm{H}, 8.63$. Found: C, 85.41; $\mathrm{H}, 8.76$. HRMS-ESI calcd for $\mathrm{C}_{20} \mathrm{H}_{24} \mathrm{NaO}$ $[\mathrm{MNa}]^{+}$303.1719, found 303.1716. 


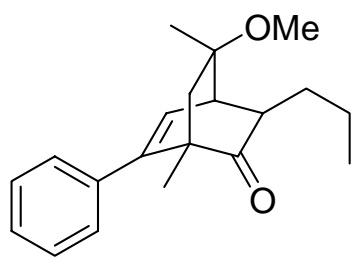

$(1 S, 3 S, 4 R, 5 R)$-5 - methoxy-1,5 - dimethyl- 7 - phenyl-3-

propylbicyclo[2.2.2] oct-7-en-2-one

$[\alpha]_{D}^{31} 157.6\left(C 1.70, \mathrm{CHCl}_{3}\right) ; \operatorname{IR}$ (neat) $v=2957,2932,2871$, 2826, 1714, 1451, 1144, 1069, 853, $764 \mathrm{~cm}^{-1} ;{ }^{1} \mathrm{H}$ NMR (300 $\left.\mathrm{MHz}, \mathrm{CDCl}_{3}\right) \delta 7.24-7.30(\mathrm{~m}, 3 \mathrm{H}), 7.01-7.04(\mathrm{~m}, 2 \mathrm{H}), 6.22(\mathrm{~d}$, $1 \mathrm{H}, \boldsymbol{J}=7.2 \mathrm{~Hz}), 3.21(\mathrm{~s}, 3 \mathrm{H}), 2.95(\mathrm{dd}, 1 \mathrm{H}, \boldsymbol{J}=6.9 \mathrm{~Hz}, \boldsymbol{J}$ $=1.9 \mathrm{~Hz}), 2.55-2.60(\mathrm{~m}, 1 \mathrm{H}), 1.87(\mathrm{~d}, 1 \mathrm{H}, \boldsymbol{J}=13.7 \mathrm{~Hz})$, $1.77-1.84(\mathrm{~m}, 1 \mathrm{H}), 1.66(\mathrm{~d}, 1 \mathrm{H}, \boldsymbol{J}=13.7 \mathrm{~Hz}), 1.33-1.49(\mathrm{~m}$, $2 \mathrm{H}), 1.35(\mathrm{~s}, 3 \mathrm{H}), 1.17-1.24(\mathrm{~m}, 1 \mathrm{H}), 1.03(\mathrm{~s}, 3 \mathrm{H}), 0.91$ $(t, 3 \mathrm{H}, \boldsymbol{J}=7.2 \mathrm{~Hz}) ;{ }^{13} \mathrm{C} \mathrm{NMR}\left(75 \mathrm{MHz}, \mathrm{CDCl}_{3}\right) \delta 213.5,144.0$, $138.1,132.1,128.2,127.9,127.2,78.7,52.8,49.5,47.1$, $44.8,42.6,34.0,25.2,21.0,16.6,14.1$; Anal. Calcd for $\mathrm{C}_{20} \mathrm{H}_{26} \mathrm{O}_{2}: \mathrm{C}, 80.50 ; \mathrm{H}, 8.78$. Found: C, 80.38; H, 9.05; HRMSEI calcd for $\mathrm{C}_{20} \mathrm{H}_{24} \mathrm{O}_{2}[\mathrm{M}]^{+}$298.1933, found 298.1931. 


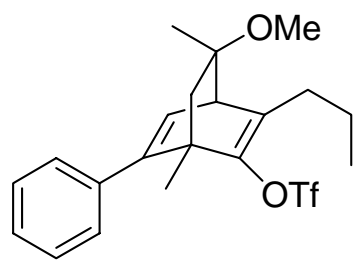

$(1 S, 4 \underline{R}, 8 R)$-8-methoxy-1, 8-dimethyl-6-phenyl-3-

propylbicyclo[2.2.2] octa-2,5-dien-2yltrifluromethanesulfonate

$[\alpha]_{D}^{34}-55.0\left(C 1.26, \mathrm{CHCl}_{3}\right) ; \operatorname{IR}$ (neat) $v=2969,1443,1399$, 1385, 1206, 1137, 1091, 1062, 1045, $862 \mathrm{~cm}^{-1} ;{ }^{1} \mathrm{H}$ NMR (300 $\left.\mathrm{MHz}, \mathrm{CDCl}_{3}\right) \delta 7.26-7.34(\mathrm{~m}, 3 \mathrm{H}), 7.08-7.11(\mathrm{~m}, 2 \mathrm{H}), 6.12(\mathrm{~d}$, $1 \mathrm{H}, J=6.5 \mathrm{~Hz}), 3.52(\mathrm{~d}, 1 \mathrm{H}, \boldsymbol{J}=6.2 \mathrm{~Hz}), 3.21(\mathrm{~s}, 3 \mathrm{H})$, $2.44-2.54(\mathrm{~m}, 1 \mathrm{H}), 2.02-2.12(\mathrm{~m}, 1 \mathrm{H}), 1.90(\mathrm{~d}, 1 \mathrm{H}, J=12.1$ $\mathrm{Hz}), 1.41-1.60(\mathrm{~m}, 2 \mathrm{H}), 1.42(\mathrm{~d}, 1 \mathrm{H}, \quad J=12.1 \mathrm{~Hz}), 1.39$ $(s, 3 \mathrm{H}), 1.29(\mathrm{~s}, 3 \mathrm{H}), 0.92(\mathrm{t}, 3 \mathrm{H}, \mathrm{J}=7.2 \mathrm{~Hz}) ;{ }^{13} \mathrm{C} \operatorname{NMR}(75$ $\left.\mathrm{MHz}, \mathrm{CDCl}_{3}\right) \delta 151.5,147.5,137.6,137.2,130.6,129.8$, 128.2, 127.8, 127.1, 83.2, 51.5, 50.4, 49.7, 47.1, 42.7, $31.7,25.0,20.4,17.5,14.0 ;$ HRMS-EI calcd for $\mathrm{C}_{17} \mathrm{H}_{17} \mathrm{~F}_{3} \mathrm{O}_{3} \mathrm{~S}$ $\left[\mathrm{M}-\mathrm{C}_{4} \mathrm{H}_{8} \mathrm{O}\right]^{+} \quad 358.0850$, found 358.0847 (Retro-Diels-Alder product) . 


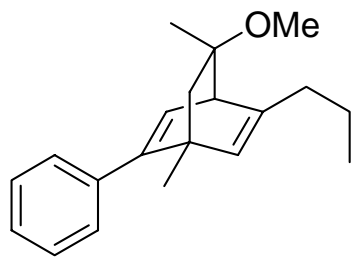

$(1 R, 4 R, 8 R)$-8-methoxy-1,8-diemthyl-2-phenyl-5-

propylbicyclo[2.2.2] octa-2,5-diene (4)

$[\alpha]_{D}^{33}-71.5\left(\mathrm{C} 0.65, \mathrm{CHCl}_{3}\right) ; \operatorname{IR}$ (neat) $v=3039,2956,2928$, $2870,2823,1596,1454,1366,1196,1158,1120,1071,865$,

$847 \mathrm{~cm}^{-1} ;{ }^{1} \mathrm{H}$ NMR $\left(300 \mathrm{MHz}, \mathrm{CDCl}_{3}\right) \delta 7.23-7.32(\mathrm{~m}, 3 \mathrm{H}), 7.05-$

$7.08(\mathrm{~m}, 2 \mathrm{H}), 6.08(\mathrm{~d}, 1 \mathrm{H}, J=6.5 \mathrm{~Hz}), 5.76(\mathrm{~s}, 1 \mathrm{H}), 3.42$

$(\mathrm{dd}, 1 \mathrm{H}, J=6.5 \mathrm{~Hz}, J=1.9 \mathrm{~Hz}), 3.23(\mathrm{~s}, 3 \mathrm{H}), 2.17-2.23$

$(\mathrm{m}, 2 \mathrm{H}), 1.41-1.55(\mathrm{~m}, 2 \mathrm{H}), 1.52(\mathrm{~d}, 1 \mathrm{H}, J=11.8 \mathrm{~Hz}), 1.43$

$(\mathrm{d}, 1 \mathrm{H}, J=12.1 \mathrm{~Hz}), 1.34(\mathrm{~s}, 3 \mathrm{H}), 1.28(\mathrm{~s}, 3 \mathrm{H}), 0.88$ (t,

$3 \mathrm{H}, \quad J=7.5 \mathrm{~Hz}) ;{ }^{13} \mathrm{C} \operatorname{NMR}\left(75 \mathrm{MHz}, \mathrm{CDCl}_{3}\right) \delta 152.1,148.1$, 139.5, 131.2, 131.0, 127.8, 127.6, 126.4, 83.8, 51.5, 50.3, 44.9, 36.7, 25.1, 22.0, 20.5, 14.0; HRMS-ESI calcd for $\mathrm{C}_{20} \mathrm{H}_{26} \mathrm{NaO}[\mathrm{MNa}]^{+}$305.1876, found 305.1873. 


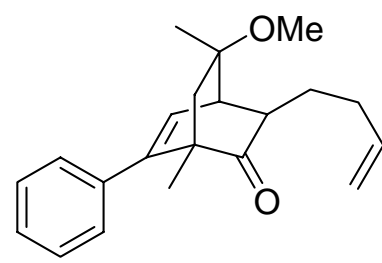

$(1 S, 3 S, 4 R, 5 R)$-3- (but - 3-enyl) -5 - methoxy-1, 5-dimethyl-7phenylbicyclo[2.2.2] oct-7-en-2-one $[\alpha]_{\mathrm{D}}^{31} 147.5$ (c 1.74, $\left.\mathrm{CHCl}_{3}\right) ; \mathrm{IR}$ (neat) $v=3016,2970,2942$, 1737, 1441, 1366, 1228, 1216, 1068, 910, $760 \mathrm{~cm}^{-1} ;{ }^{1} \mathrm{H}$ NMR (300 $\left.\mathrm{MHz}, \mathrm{CDCl}_{3}\right) \delta 7.26-7.32(\mathrm{~m}, 3 \mathrm{H}), 7.02-7.06(\mathrm{~m}, 2 \mathrm{H})$, $6.25(\mathrm{~d}, 1 \mathrm{H}, \boldsymbol{J}=6.9 \mathrm{~Hz}), 5.77-5.86(\mathrm{~m}, 1 \mathrm{H}), 5.00-5.09(\mathrm{~m}$, $2 \mathrm{H}), 3.22(\mathrm{~s}, 3 \mathrm{H}), 2.97(\mathrm{dd}, 1 \mathrm{H}, \boldsymbol{J}=6.9 \mathrm{~Hz}, \boldsymbol{J}=1.9 \mathrm{~Hz})$, $2.58-2.63(\mathrm{~m}, 1 \mathrm{H}), 2.14-2.22(\mathrm{~m}, 2 \mathrm{H}), 1.92-1.96(\mathrm{~m}, 1 \mathrm{H})$, $1.88(\mathrm{~d}, 1 \mathrm{H}, J=14.0 \mathrm{~Hz}), 1.67(\mathrm{~d}, 1 \mathrm{H}, J=13.7 \mathrm{~Hz}), 1.37$ $(\mathrm{s}, 3 \mathrm{H}), 1.18-1.40(\mathrm{~m}, 1 \mathrm{H}), 1.04(\mathrm{~s}, 3 \mathrm{H}) ;{ }^{13} \mathrm{C} \mathrm{NMR}(75 \mathrm{MHz}$, $\left.\mathrm{CDCl}_{3}\right) \delta 213.6,152.9,144.4,138.4,132.2,128.4,128.1$, 127.5, 115.3, 78.9, 53.0, 49.8, 47.4, 45.0, 42.4, 32.2, 31.3, 25.4, 16.8; Anal. Calcd for $\mathrm{C}_{21} \mathrm{H}_{26} \mathrm{O}_{2}: \mathrm{C}, 81.25$; $\mathrm{H}$, 8.44. Found: $\mathrm{C}, 81.18 ; \mathrm{H}, 8.35$; HRMS-EI calcd for $\mathrm{C}_{21} \mathrm{H}_{26} \mathrm{O}_{2}$ $[\mathrm{M}]^{+} 310.1933$, found 310.1929 . 


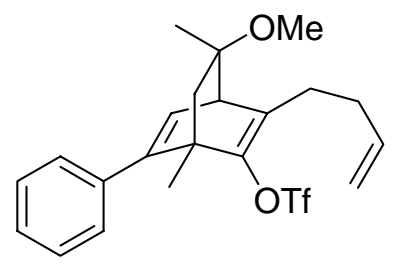

$(1 S, 4 R, 8 R)$-3-( but - 3-enyl) -8-methoxy-1, 8-dimethyl-6 -

phenylbicyclo[2.2.2] octa-2,5-dien-2yltrifluromethanesulfonate

$[\alpha]_{\mathrm{D}}^{34}-70.9\left(\boldsymbol{c} 1.44, \mathrm{CHCl}_{3}\right) ; \operatorname{IR}$ (neat) $v=2976,2827$, $1684,1641,1399,1205,1138,1087,1046,861 \mathrm{~cm}^{-1} ;{ }^{1} \mathrm{H}$ NMR (300 $\left.\mathrm{MHz}, \mathrm{CDCl}_{3}\right) \delta 7.26-7.35(\mathrm{~m}, 3 \mathrm{H}), 7.07-7.11(\mathrm{~m}, 2 \mathrm{H})$, $6.11(\mathrm{~d}, 1 \mathrm{H}, \mathrm{J}=6.2 \mathrm{~Hz}), 5.78-5.83(\mathrm{~m}, 1 \mathrm{H}), 4.96-5.07(\mathrm{~m}$, $2 \mathrm{H}), 3.53(\mathrm{~d}, 1 \mathrm{H}, \boldsymbol{J}=6.2 \mathrm{~Hz}), 3.21(\mathrm{~s}, 3 \mathrm{H}), 2.58-2.64(\mathrm{~m}$, $1 \mathrm{H}), 2.16-2.30(\mathrm{~m}, 3 \mathrm{H}), 1.90(\mathrm{~d}, 1 \mathrm{H}, \boldsymbol{J}=12.1 \mathrm{~Hz}), 1.42(\mathrm{~d}$, $1 \mathrm{H}, \quad J=12.1 \mathrm{~Hz}), 1.39(\mathrm{~s}, 3 \mathrm{H}), 1.29(\mathrm{~s}, 3 \mathrm{H}) ;{ }^{13} \mathrm{C} \mathrm{NMR}(75$ $\left.\mathrm{MHz}, \mathrm{CDCl}_{3}\right) \delta 151.4,147.6,137.6,137.5,136.6,130.6$, 128.2, 127.8, 127.1, 115.1, 83.2, 51.8, 50.4, 49.6, 47.1, 31.3, 29.3, 25.0, 17.4; Anal. Calcd for $\mathrm{C}_{22} \mathrm{H}_{25} \mathrm{~F}_{3} \mathrm{O}_{4} \mathrm{~S}: \mathrm{C}$, 59.72; H, 5.69. Found: C, 59.83; H, 5.83. HRMS-ESI calcd for $\mathrm{C}_{22} \mathrm{H}_{25} \mathrm{~F}_{3} \mathrm{NaO}_{4} \mathrm{~S}$ [MNa] ${ }^{+} 465.1318$, found 465.1325 . 


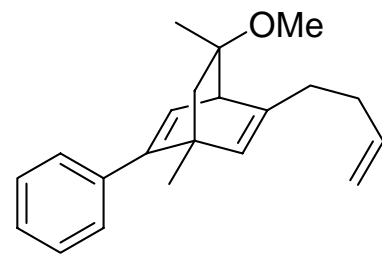

$(1 R, 4 R, 8 R)$ - 5- (but - 3-enyl) - 8-methoxy - 1, 8 -dimethyl-2 -

phenylbicyclo[2.2.2]-octa-2,5-diene (5)

$[\alpha]_{\mathrm{D}}^{30}-53.4\left(\mathrm{C} 0.19, \mathrm{CHCl}_{3}\right) ; \operatorname{IR}$ (neat) $v=2927,1457,1213$, 1073, $864 \mathrm{~cm}^{-1} ;{ }^{1} \mathrm{H} \mathrm{NMR}\left(300 \mathrm{MHz}, \mathrm{CDCl}_{3}\right) \delta 7.23-7.31(\mathrm{~m}, 3 \mathrm{H})$, $7.04-7.11(\mathrm{~m}, 2 \mathrm{H}), 6.08(\mathrm{~d}, 1 \mathrm{H}, \boldsymbol{J}=6.3 \mathrm{~Hz}), 5.78-5.90(\mathrm{~m}$, $2 \mathrm{H}), 4.93-5.05(\mathrm{~m}, 2 \mathrm{H}), 3.44(\mathrm{dd}, 1 \mathrm{H}, \boldsymbol{J}=6.7 \mathrm{~Hz}, \boldsymbol{J}=1.9$ $\mathrm{Hz}), 3.22(\mathrm{~s}, 3 \mathrm{H}), 2.22-2.34(\mathrm{~m}, 3 \mathrm{H}), 1.38-1.50(\mathrm{~m}, 3 \mathrm{H})$, $1.33(\mathrm{~s}, 3 \mathrm{H}), 1.28(\mathrm{~s}, 3 \mathrm{H}) ;{ }^{13} \mathrm{C} \mathrm{NMR}\left(75 \mathrm{MHz}, \mathrm{CDCl}_{3}\right) \delta 147.6$, 139.5, 138.5, 131.2, 131.1, 128.2, 127.8, 127.6, 126.4, $114.4,83.8,51.8,50.6,50.3,45.0,34.1,31.7,25.2$,

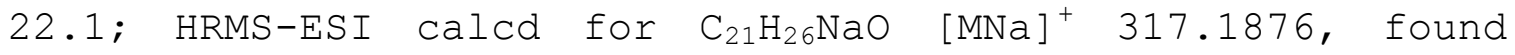
317.1871.

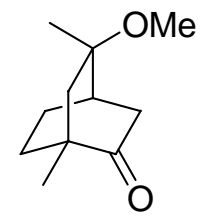

$(1 R, 4 R, 5 R)$-5-methoxy-1,5-diemthylbicyclo[2.2.2] octan-2-one $[\alpha]_{\mathrm{D}}^{30} 1.5\left(\mathrm{C} 1.02, \mathrm{CHCl}_{3}\right) ; \operatorname{IR}$ (film) $v=2927,2826,1721$, 1454, 1375, 1220, 1174, 1085, $772 \mathrm{~cm}^{-1} ;{ }^{1} \mathrm{H}$ NMR $(300 \mathrm{MHz}$, $\left.\mathrm{CDCl}_{3}\right) \delta 3.13(\mathrm{~s}, 3 \mathrm{H}), 2.62(\mathrm{dt}, 1 \mathrm{H}, \mathrm{J}=18.6 \mathrm{~Hz}, \boldsymbol{J}=2.9$ 
$\mathrm{Hz}), 2.18(\mathrm{t}, 1 \mathrm{H}, \boldsymbol{J}=2.9 \mathrm{~Hz}), 1.98(\mathrm{dd}, 1 \mathrm{H}, \boldsymbol{J}=18.6 \mathrm{~Hz}, \boldsymbol{J}$ $=2.8 \mathrm{~Hz}), 1.50-1.86(\mathrm{~m}, 6 \mathrm{H}), 1.33(\mathrm{~s}, 3 \mathrm{H}), 0.91(\mathrm{~s}, 3 \mathrm{H})$; ${ }^{13} \mathrm{C} \quad \mathrm{NMR} \quad\left(75 \mathrm{MHz}, \mathrm{CDCl}_{3}\right) \delta 216.4,75.2,48.9,47.1,44.0$, 39.7, 36.6, 29.9, 23.2, 22.3, 19.9; HRMS-EI calcd for $\mathrm{C}_{11} \mathrm{H}_{18} \mathrm{O}_{2}[\mathrm{M}]^{+}$182.1307, found 182.1302 .

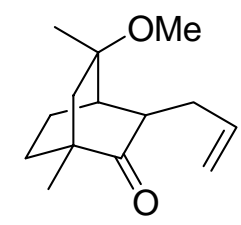

$(1 R, 3 S, 4 R, 5 R)-3$ - allyl-5-methoxy-1, 5dimethylbicyclo[2.2.2]octan-2-one

$[\alpha]_{\mathrm{D}}^{24} 5.3\left(\mathrm{C} 0.57, \mathrm{CHCl}_{3}\right) ; \operatorname{IR}(\mathrm{film}) \mathrm{v}=2927,1718$, 1640, 1457, 1375, $1067 \mathrm{~cm}^{-1} ;{ }^{1} \mathrm{H} \operatorname{NMR}\left(300 \mathrm{MHz}, \mathrm{CDCl}_{3}\right) \quad \delta \quad 5.69-5.82$ $(\mathrm{m}, 1 \mathrm{H}), 5.00-5.13(\mathrm{~m}, 2 \mathrm{H}), 3.10(\mathrm{~s}, 3 \mathrm{H}), 2.64-2.72(\mathrm{~m}$, $2 \mathrm{H}), \quad 1.86-2.09(\mathrm{~m}, 2 \mathrm{H}), 1.40-1.79(\mathrm{~m}, 6 \mathrm{H}), 1.36(\mathrm{~s}, 3 \mathrm{H})$, $0.90(\mathrm{~s}, 3 \mathrm{H}) ;{ }^{13} \mathrm{C} \mathrm{NMR}\left(75 \mathrm{MHz}, \mathrm{CDCl}_{3}\right) \delta 218.0,136.7,116.4$, $75.3,48.7,46.7,45.4,44.5,38.0,31.5,30.6,23.7,19.9$,

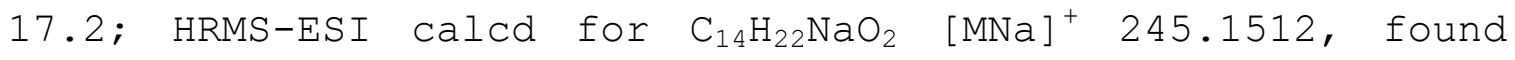
245.1509 . 


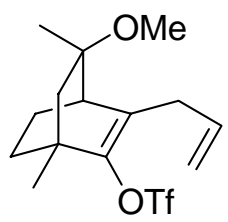

$(1 R, 4 R, 5 R)$-3-allyl-5-methoxy-1,5-dimethylbicyclo[2.2.2] oct 2-en-2-yltrifluoromethanesulfonate $[\alpha]_{\mathrm{D}}^{26} 64.7$ ( $\left.\mathrm{C} 0.78, \mathrm{CHCl}_{3}\right) ; \operatorname{IR~(film)~} v=2943,2823$, 1401, 1209, 1140, $881 \mathrm{~cm}^{-1} ;{ }^{1} \mathrm{H} \mathrm{NMR}\left(300 \mathrm{MHz}, \mathrm{CDCl}_{3}\right) \delta 5.63-5.79$ (m, $1 \mathrm{H}), 5.07-5.18(\mathrm{~m}, 2 \mathrm{H}), 3.28-3.50(\mathrm{~m}, 1 \mathrm{H}), 3.24(\mathrm{dt}, 1 \mathrm{H}, \mathrm{J}$ $=5.7 \mathrm{~Hz}, \boldsymbol{J}=1.5 \mathrm{~Hz}), 3.09(\mathrm{~s}, 3 \mathrm{H}), 2.79(\mathrm{~m}, 1 \mathrm{H}), 2.71$ (t, $1 \mathrm{H}, \boldsymbol{J}=2.8 \mathrm{~Hz}), 1.64-1.73(\mathrm{~m}, 2 \mathrm{H}), 1.42-1.53(\mathrm{~m}, 1 \mathrm{H}), 1.28$ $(\mathrm{s}, 3 \mathrm{H}), 1.19(\mathrm{~s}, 3 \mathrm{H}), 1.17-1.34(\mathrm{~m}, 2 \mathrm{H}) ;{ }^{13} \mathrm{C} \mathrm{NMR}(75 \mathrm{MHz}$, $\left.\mathrm{CDCl}_{3}\right) \delta 143.7,133.9,133.2,117.4,78.7,50.8,49.0,45.0$, 42.7, 34.5, 32.8, 23.2, 22.7, 20.2; Anal. Calcd for $\mathrm{C}_{15} \mathrm{H}_{21} \mathrm{~F}_{3} \mathrm{O}_{4} \mathrm{~S}: \mathrm{C}, 50.84 ; \mathrm{H}, 5.97$. Found: C, 50.62; H, 5.98; HRMS-ESI calcd for $\mathrm{C}_{15} \mathrm{H}_{21} \mathrm{~F}_{3} \mathrm{O}_{4} \mathrm{NaS} \quad[\mathrm{MNa}]^{+} 377.1005$, found 377.1000

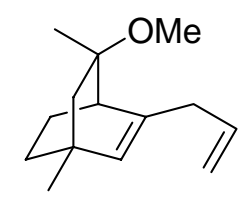

$(1 R, 4 R, 5 R)$-3-allyl-5-methoxy-1,5-dimethylbicyclo[2.2.2] oct 2-ene (12) $[\alpha]_{\mathrm{D}}^{28} 95.4\left(\mathrm{C} 0.67, \mathrm{CHCl}_{3}\right) ; \operatorname{IR}$ (film) $v=2947,1637,1456$, 1369, 1120, 1075, $994 \mathrm{~cm}^{-1} ;{ }^{1} \mathrm{H}$ NMR (300 $\left.\mathrm{MHz}, \mathrm{CDCl}_{3}\right) \delta 5.78-$ 
$5.89(\mathrm{~m}, 1 \mathrm{H}), 5.58(\mathrm{~s}, 1 \mathrm{H}), 5.00-5.12(\mathrm{~m}, 2 \mathrm{H}), 3.14$ ( $\mathrm{s}$, $3 \mathrm{H}), \quad 1.84-2.09(\mathrm{~m}, 2 \mathrm{H}), 1.57-1.68(\mathrm{~m}, 1 \mathrm{H}), 1.29(\mathrm{~s}, 3 \mathrm{H})$,

$1.09-1.35(\mathrm{~m}, 5 \mathrm{H}), 1.08(\mathrm{~s}, 3 \mathrm{H}) ;{ }^{13} \mathrm{C} \mathrm{NMR}\left(75 \mathrm{MHz}, \mathrm{CDCl}_{3}\right) \delta$ $143.6,136.1,129.1,115.7,79.1,50.9,49.2,42.6,40.1$, $35.6,32.3,25.2,23.8,23.6$; Anal. Calcd for $\mathrm{C}_{14} \mathrm{H}_{22} \mathrm{O}$ : C, 81.50; H, 10.75. Found: C, 81.23; H, 10.72; HRMS-ESI calcd for $\mathrm{C}_{14} \mathrm{H}_{22} \mathrm{NaO}[\mathrm{MNa}]^{+} 229.1563$, found 245.1559 .

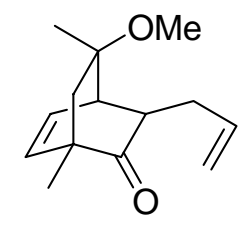

$(1 S, 3 S, 4 R, 5 R)-3-a l l y l-5-$ methoxy -1, 5 dimethylbicyclo[2.2.2] oct-7-en-2-one

$[\alpha]_{\mathrm{D}}^{25}-275.5$ (c $\left.0.78, \mathrm{CHCl}_{3}\right) ; \operatorname{IR}$ (film) $v=2972,2930$, $1720,1640,1449,1080 \mathrm{~cm}^{-1} ;{ }^{1} \mathrm{H} \mathrm{NMR}\left(300 \mathrm{MHz}, \mathrm{CDCl}_{3}\right) \delta 6.34$ $(t, 1 \mathrm{H}, J=6.8 \mathrm{~Hz}), 5.70-5.84(\mathrm{~m}, 2 \mathrm{H}), 5.00-5.06(\mathrm{~m}, 2 \mathrm{H})$, $3.16(\mathrm{~s}, 3 \mathrm{H}), 2.86(\mathrm{~d}, 1, \mathrm{~J}=6.8 \mathrm{~Hz}), 2.53-2.62(\mathrm{~m}, 2 \mathrm{H})$, $1.78(\mathrm{~d}, 1 \mathrm{H}, \boldsymbol{J}=13.7 \mathrm{~Hz}), 1.75-1.81(\mathrm{~m}, 1 \mathrm{H}), 1.47(\mathrm{~d}, 1 \mathrm{H}$, $J=13.7), 1.25(\mathrm{~s}, 3 \mathrm{H}), 1.17(\mathrm{~s}, 3 \mathrm{H}) ;{ }^{13} \mathrm{C} \mathrm{NMR}(75 \mathrm{MHz}$, $\left.\mathrm{CDCl}_{3}\right) \delta 212.5,136.5,134.6,133.2,116.3,78.9,50.5$, $49.5,45.9,44.4,42.4,36.0,25.0,17.4 ;$ HRMS-EI calcd for $\mathrm{C}_{14} \mathrm{H}_{20} \mathrm{O}[\mathrm{M}]^{+} 220.1463$, found 220.1460 . 


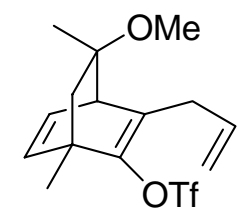

$(1 S, 4 R, 5 R)-3-a l l y l-5-$ methoxy -1, 5-

dimethylbicyclo[2,2.2] octa-2,7-dien-2-

\section{yltrifluoromethanesulfonate}

$[\alpha]_{\mathrm{D}}^{27} 44.4\left(\mathrm{C} 0.65, \mathrm{CHCl}_{3}\right) ; \operatorname{IR}(\mathrm{film}) v=2928,2931,2825$, 1403, 1210, 1141, $869 \mathrm{~cm}^{-1} ;{ }^{1} \mathrm{H}$ NMR (300 $\left.\mathrm{MHz}, \mathrm{CDCl}_{3}\right) \delta 6.27$ (t, $1 \mathrm{H}, \boldsymbol{J}=6.2 \mathrm{~Hz}), 6.10(\mathrm{dd}, 1 \mathrm{H}, \boldsymbol{J}=7.2 \mathrm{~Hz}, \boldsymbol{J}=1.6 \mathrm{~Hz})$, $5.64-5.77(\mathrm{~m}, 1 \mathrm{H}), 5.00-5.10(\mathrm{~m}, 2 \mathrm{H}), 3.42(\mathrm{dd}, 1 \mathrm{H}, \quad \boldsymbol{J}=$ $6.2 \mathrm{~Hz}, J=1.6 \mathrm{~Hz}), 3.23-3.31(\mathrm{~m}, 1 \mathrm{H}), 3,16(\mathrm{~s}, 3 \mathrm{H}), 2.71-$ $2.78(\mathrm{~m}, 1 \mathrm{H}), 1.84(\mathrm{~d}, 1 \mathrm{H}, \boldsymbol{J}=11.8 \mathrm{~Hz}), 1.47(\mathrm{~s}, 3 \mathrm{H}), 1.25$ $(\mathrm{s}, 3 \mathrm{H}), 1.23(\mathrm{~d}, 1 \mathrm{H}, \boldsymbol{J}=11.8 \mathrm{~Hz}) ;{ }^{13} \mathrm{C} \mathrm{NMR}\left(75 \mathrm{MHz}, \mathrm{CDCl}_{3}\right) \delta$ $147.8,140.7,133.9,133.7,133.3,120.5,117.1,83.5$, $51.7,50.3,48.6,44.7,34.1,24.7,17.9$; Anal. Calcd for $\mathrm{C}_{15} \mathrm{H}_{19} \mathrm{~F}_{3} \mathrm{O}_{4} \mathrm{~S}: \mathrm{C}, 51.13 ; \mathrm{H}$, 5.43. Found: C, 51.34; $\mathrm{H}, 5.58$; HRMS-ESI calcd for $\mathrm{C}_{15} \mathrm{H}_{19} \mathrm{~F}_{3} \mathrm{O}_{4} \mathrm{NaS} \quad[\mathrm{MNa}]^{+} 375.0848$, found 375.0842 . 


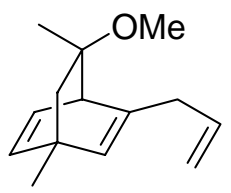

$(1 S, 4 R, 5 R)-3-a l l y l-5-$ methoxy -1, 5 dimethylbicyclo[2.2.2] octa-2,7-diene (13)

$[\alpha]_{\mathrm{D}}^{33} 64.3\left(\mathrm{C} 0.45, \mathrm{CHCl}_{3}\right) ; \mathrm{IR}$ (film) $v=2954,2926,2823$, 1456, 1365, 1331, 1101, $1072 \mathrm{~cm}^{-1} ;{ }^{1} \mathrm{H} \mathrm{NMR}\left(300 \mathrm{MHz}, \mathrm{CDCl}_{3}\right) \delta$ $6.26(t, 1 \mathrm{H}, J=6.9 \mathrm{~Hz}), 6.07(\mathrm{~d}, 1 \mathrm{H}, \boldsymbol{J}=7.2 \mathrm{~Hz}), 5.72-$ $5.87(\mathrm{~m}, 2 \mathrm{H}), 5.00-5.06(\mathrm{~m}, \mathrm{H}), 3.36(\mathrm{dd}, 1 \mathrm{H}, \boldsymbol{J}=6.2 \mathrm{~Hz}, \boldsymbol{J}$ $=1.5 \mathrm{~Hz}), 3.18(\mathrm{~s}, 3 \mathrm{H}), 2.81-3.17(\mathrm{~m}, 2 \mathrm{H}), 1.40(\mathrm{~s}, 3 \mathrm{H})$, $1.40(\mathrm{~d}, 1 \mathrm{H}, \boldsymbol{J}=11.8 \mathrm{~Hz}), 1.22(\mathrm{~s}, 3 \mathrm{H}), 1.19(\mathrm{~d}, 1 \mathrm{H}, \boldsymbol{J}=$ $11.8 \mathrm{~Hz}) ;{ }^{13} \mathrm{C} \operatorname{NMR}\left(75 \mathrm{MHz}, \mathrm{CDCl}_{3}\right) \delta 145.9,141.7,136.0$, 133.4, 131.7, 115.9, 84.0, 51.2, 50.2, 49.5, 42.6, 39.1, 24.7, 22.2; Anal. Calcd for $\mathrm{C}_{14} \mathrm{H}_{20} \mathrm{O}: \mathrm{C}, 82.30 ; \mathrm{H}, 9.87$. Found: C, 82.37; H, 9.81.

\section{References}

(1) Takaya, Y.; Ogasawara, M.; Hayashi, T. Tetrahedron Lett. 1999, 40, 6957.

(2) Itooka, R.; Iguchi, Y.; Miyaura, N. J. org Chem. 2003, 68, 6000 .

(3) Boiteau, J.-G.; Imbos, R.; Minnaard, A. J.; Feringa, B. L. Org. Lett. 2003, 5, 691 and 1385. 
(4) Chaigne, F.; Gotteland, J.-P.; Malacria, M. Tetrahedron Lett. 1989, 30, 1803.

(5) Patro, B.; Deb, B.; Ila, H.; Junjappa, H. J. Org. Chem. 1992, 57, 2257.

(6) Denmark, S. E.; Amishiro, N. J. Org. Chem. 2003, 68, 6997.

(7) Dawson, G. J.; Williams, J. M. J.; Coote, S. J. Tetrahedron: Asymmetric 1995, 6, 2535.

(8) Meyers, A. I.; Whitten, C. E. Tetrahedron Lett. 1976, 23, 1947 .

(9) Majumder, P. L.; Chatterjee, S.; Mukhoti, N. J. Indian Chem. Soc. 2001, 78, 743.

(10) Oi, S.; Moro, M.; Ito, H.; Honma, Y.; Miyano, S.; Inoue, Y. Tetrahedron 2002, 58, 91.

(11) Hayashi, T.; Yamamoto, A.; Hagihara, T. J. org. Chem. 1986, 51, 723.

(12) Humphrey, C. E.; Turner, N. J.; Easson, M. A. M.; Flitsch, S. L.; Ulijn, R. V. J. Am. Chem. Soc. 2003, 125,13952 .

(13) Amberg, W.; Seebach, D. Chem. Ber. 1990, 123, 2413. 